\title{
Mimicking Portfolios, Economic Risk Premia, and Tests of Multi-beta Models \\ by
}

Pierluigi Balduzzi* and Cesare Robotti**

This draft: November 2006.

\begin{abstract}
We consider two formulations of the linear factor model with non-traded factors. In the first formulation (LFM), risk premia and alphas are estimated by a cross-sectional regression of average returns on betas. In the second formulation $\left(\mathrm{LFM}^{\star}\right)$, the factors are replaced by their projections on the span of excess returns, and risk premia and alphas are estimated by time-series regressions. We compare the two formulations and study the small-sample properties of estimates and test statistics. We conclude that the $\mathrm{LFM}^{\star}$ formulation should be considered in addition to, or even instead of, the more traditional LFM formulation.

JEL \# G12
\end{abstract}

* Boston College. ${ }^{* *}$ Federal Reserve Bank of Atlanta. Corresponding author: Pierluigi Balduzzi, Boston College, Finance Department, The Wallace E. Carroll School of Management, 438 Fulton Hall, 140 Commonwealth Ave., Chestnut Hill, MA 02467. E-mail: balduzzp@bc.edu. Tel.: 617-552-3976. The authors thank Torben Andersen, the Editor, an anonymous Associate Editor, and an anonymous referee for valuable comments. The authors thank Raymond Kan, Wayne Ferson, Jay Shanken, and Dan Waggoner for several useful conversations and comments. The authors also thank seminar participants at the 2004 All Georgia Finance Conference for useful comments. 


\title{
Mimicking Portfolios, Economic Risk Premia, and Tests of Multi-beta Models
}

\begin{abstract}
We consider two formulations of the linear factor model with non-traded factors. In the first formulation (LFM), risk premia and alphas are estimated by a cross-sectional regression of average returns on betas. In the second formulation $\left(\mathrm{LFM}^{\star}\right)$, the factors are replaced by their projections on the span of excess returns, and risk premia and alphas are estimated by time-series regressions. We compare the two formulations and study the small-sample properties of estimates and test statistics. We conclude that the $\mathrm{LFM}^{\star}$ formulation should be considered in addition to, or even instead of, the more traditional LFM formulation.

JEL \# G12
\end{abstract}




\section{Introduction}

Most asset-pricing models explain the cross-section of expected returns in terms of exposures, or betas, to one or more risk factors. We denote these models as multi-beta models. In the case of the Consumption-based Capital Asset Pricing Model (C-CAPM) and the Intertemporal Capital Asset Pricing Model (I-CAPM), one or more of the risk factors are "economic," i.e. they are not themselves asset returns. The standard formulation of such asset-pricing models starts from a linear factor model, LFM

$$
r_{t}=\alpha+\beta^{\top}\left[\lambda+y_{t}-E\left(y_{t}\right)\right]+e_{t}
$$

where $r_{t}$ is an $N \times 1$ vector of returns in excess of the risk-free rate, $\alpha$ is the vector of deviations from the model, $\lambda$ is the vector of economic risk premia, $y_{t}$ is a $K \times 1$ vector of factor realizations, and $e_{t}$ is an $N \times 1$ vector of residuals orthogonal to the factors. If the multi-beta model is correct, then $\alpha=0$, and any portfolio with a beta of one w.r.t. factor $y_{k}$ and zero beta w.r.t. all the other factors, a unit-beta portfolio, earns a risk premium equal to $\lambda_{k}$.

The risk premia on the unit-beta portfolios can be immediately estimated as the coefficients (or averages of coefficients) of a cross-sectional regression (CSR) of average returns (or returns) on betas. This CSR can be performed using a variety of weighting schemes. We focus on two schemes. In the first scheme the assets are unweighted. This corresponds to a CSR OLS-style, following the seminal articles by Black, Jensen, and Scholes (1972) and Fama and MacBeth (1973). This approach has been implemented in a large number of empirical studies. In the second scheme, the assets are weighted by the inverse of the covariance matrix of the idiosyncratic components. This corresponds to a CSR GLS-style regression.

An alternative formulation of the LFM obtains when we replace the factors with the variable component of their projections onto the span of excess returns, augmented with a constant (see Huberman, Kandel, and Stambaugh, 1987). We have

$$
y_{t}=\gamma_{0}^{\star}+\left(\gamma^{\star}\right)^{\top} r_{t}+\epsilon_{t}
$$

and $y_{t}^{\star} \equiv\left(\gamma^{\star}\right)^{\top} r_{t}$, where $\gamma^{\star}=\Sigma_{r r}^{-1} \Sigma_{r y}$. For the mimicking portfolios, $y_{t}^{*}$, to exist we assume 
that $\left(\gamma^{\star}\right)^{\top} 1_{N}=\Sigma_{y r} \Sigma_{r r}^{-1} 1_{N} \neq 0_{K}$, where $1_{N}$ is an $N \times 1$ vector of ones and $0_{K}$ is a $K \times 1$ vector of zeros. This condition is equivalent to assuming that the global minimum-variance portfolio has positive systematic risk. We then have

$$
r_{t}=\alpha^{\star}+\left(\beta^{\star}\right)^{\top} y_{t}^{\star}+e_{t}^{\star}
$$

We denote this alternative formulation as $\mathrm{LFM}^{\star}$.

The projection coefficients are the weights of portfolios whose returns have maximum (squared) correlations with the factors. Hence, these maximum-correlation portfolio weights are proportional to the hedging-portfolio weights of Merton (1973). Other examples of this second approach are Breeden (1979), Breeden, Gibbons, and Litzenberger (1989), Fama (1996), Lamont (2001), and Ferson, Siegel, and Xu (2005).

Table I presents results for the LFM and the LFM ${ }^{\star}$ formulations of the C-CAPM, and of I-CAPM where the non-traded risk factor is the dividend yield. (Details on the estimation procedures and the data used can be found later on in the paper.) Table I shows how, depending on the linear factor model, parameter estimates and statistical inference can differ substantially. For example, in the case of the C-CAPM, the consumption risk premium is 0.2410 and significant in the LFM (CSR-OLS), but only 0.0062 and insignificant in the $\mathrm{LFM}^{\star}$. Moreover, the C-CAPM is strongly rejected in the $\mathrm{LFM}^{\star}$ formulation, but not in the LFM formulation, CSR-OLS version. Given the difference in results, it is obvious to ask which representation is most likely to deliver the correct estimates and inference. The existing literature does not provide an answer to this question.

Our small-sample analysis should help the researcher draw the correct conclusions, when facing different results from different methods (more on our simulation results below). For example, we show that the percentage bias in the risk-premium estimates for the C-CAPM is more pronounced in the case of the CSR-OLS estimates, than for the $\lambda^{\star}$ estimates. At the same time, though, the $\mathrm{LFM}^{\star}$ formulation leads to over-rejections of the model under the null, while the LFM representation has roughly the correct size. Hence, an econometrician faced with the results above should be more (less) inclined to trust the estimates (test statistics) resulting from the $\mathrm{LFM}^{\star}$ representation than from the LFM representation.

We start our analysis by clarifying the relation between the LFM and LFM ${ }^{\star}$ formulations, 
and the relation between the unit-beta and maximum-correlation portfolios. In doing so, we provide new insights relative to the existing literature. For example, we show that the alphas resulting from the CSR-GLS estimation of the LFM are the same as the alphas in the LFM* formulation. We also show that the maximum Sharpe ratio attainable from the CSR-GLS unit-beta portfolios is the same as that attainable from the maximum-correlation portfolios.

Following Hansen (1982) and Cochrane (2001), we show how both formulations of the linear factor model, and both estimation approaches (CSR vs time-series), can be cast within the unifying framework of GMM. This approach allows us to derive the asymptotic properties of estimates and statistics without the need for restrictive distributional assumptions. We then construct a simulation exercise to assess the properties of the estimators for the two different formulations of the model. The simulations consider a one-factor (C-CAPM) and a two-factor (I-CAPM) economy, both i.i.d. and serially-correlated data, different lengths of the sample (525 vs 240 months), and different choices of test assets (ten size-sorted portfolios vs the 25 size- and value-sorted portfolios of Fama and French, 1993).

In summary, in our simulation exercises the $\mathrm{LFM}^{\star}$ formulation performs better than the LFM-CSR-GLS formulation, and similarly to the LFM-CSR-OLS formulation, in terms of bias of the risk premium estimates. In terms of RMSEs of the estimates and power of the tests, the LFM ${ }^{\star}$ and LFM-CSR-GLS perform similarly, and they perform better than the LFM-CSR-OLS formulation. In the case of a noisy factor, the LFM $^{*}$ formulation works much better than either LFM formulation. These results lead us to conclude that the LFM* formulation should be considered in addition to, or even instead of, the more traditional LFM formulation.

The paper is organized as follows: Section I provides a review of the literature. Section II illustrates the two alternative formulations of a linear factor model. Section III discusses the properties of the unit-beta and maximum-correlation mimicking portfolios, and derives the maximum Sharpe ratios obtainable from the different types of mimicking portfolios. Section IV derives the moment conditions used in estimation. Section V derives the asymptotic properties of the GMM estimators. Section VI discusses the case where both traded and nontraded factors are present, and where the non-traded factor is observed with noise. Section VII illustrates the data used to calibrate the simulation exercise. Section VIII describes the 
bootstrap experiment. Section IX discusses the results of the simulation. Section X explores some extensions of the analysis. The final section concludes our findings and the Appendix contains proofs of the analytical results.

\section{Related literature}

Several papers have studied the properties of CSR risk-premium estimators. Litzenberger and Ramaswamy (1979), for example, document the error-in-variables (EIV) problem arising in this setting, and suggest a correction that asymptotically removes the resulting bias. Amsler and Schmidt (1985) and MacKinlay (1987), in comparison, perform Monte Carlo exercises focusing on the small-sample properties of test statistics of the CAPM. Shanken (1992) considers the properties of the CSR estimator both when the time dimension is large, and when the cross-sectional dimension is large. Shanken and Zhou (2006) focus on the small sample properties of alternative CSR risk-premium estimators. Affleck-Graves and Bradfield (1993) perform a simulation exercise to assess the power of the CSR estimator in rejecting the hypothesis of a zero market-risk premium, when the simulated economy has a positive market premium. Kim (1995) provides an asymptotic correction for the EIV problem, which is robust to conditional heteroskedasticity. More recently, Chen and Kan (2006) develop finite-sample corrections for the EIV bias.

Other studies have focused on the comparison between stochastic discount factor (SDF) and LFM representations in tests of multi-beta models. Cochrane (2001) focuses on tests with a traded factor (the excess return on the market). Kan and Zhou (1999, 2001a) and Jagannathan and Wang (2002), on the other hand, consider the case where the factor is not traded.

Closely related to this paper is Kimmel (2003). Kimmel derives the asymptotic properties of estimates of risk premia on CSR-GLS unit-beta portfolios and on maximum-correlation portfolios. Kimmel's paper differs from ours because he focuses on asymptotics only, for the

case of Gaussian i.i.d. returns, and because he does not address issues of size and power of the test statistics.

Also closely related to this paper is Asgharian (2004). Asgharian shows how the choice 
of base assets affects the properties of the returns on maximum-correlation portfolios. He also shows how testing a multi-beta model in its LFM or LFM ${ }^{\star}$ formulation can lead to different conclusions. In addition, Asgharian discusses the "portfolio" method of estimating factor realizations, where assets with a high loading on a factor receive a positive weight, while assets with a low loading on a factor receive a negative weight (see, for example, Chan et al., 1998, and Fama and French, 1993). He points out how the portfolio method may lead to weights that are quite different from the CSR portfolio weights, and suggests an alternative method based on the relative differences in the loadings. Asgharian's paper differs from ours because he does not study the theoretical relation between the LFM and LFM* representations, and because he does not compare the small-sample properties of estimates and test statistics for the two formulations.

Finally, Asgharian and Hansson (2005) consider the effects of replacing the original factors in the LFM with the factor realizations estimated using the portfolio method, using an equally-weighted scheme. They show that the betas and alphas change considerably. Their paper differs from ours because they focus on the implications of the portfolio method, rather than on the properties of the LFM and $\mathrm{LFM}^{\star}$ representations.

\section{Relation between LFM and $\mathrm{LFM}^{\star}$}

The next result establishes the relation between the two representations.

Result 1. Mispricing in the $\mathrm{LFM}^{\star}$ representation relates to mispricing in the LFM representation as follows

$$
\alpha^{\star}=\left[I-\left(\beta^{\star}\right)^{\top}\left(\gamma^{\star}\right)^{\top}\right] \alpha
$$

where $\left[I-\left(\beta^{\star}\right)^{\top}\left(\gamma^{\star}\right)^{\top}\right]$ is of rank $N-K$ with null space given by $\beta^{\top} \delta, \delta$ being a generic real-valued $N \times 1$ vector.

Hence, if $\alpha=0, \alpha^{\star}=0$. On the other hand, if $\alpha^{\star}=0, \alpha$ is not necessarily zero, since the $N \times N$ matrix $\left[I-\left(\beta^{\star}\right)^{\top}\left(\gamma^{\star}\right)^{\top}\right]$ is of rank $N-K$. Yet, the only case where $\alpha^{\star}=0$, but $\alpha \neq 0$, is when $\alpha=\beta^{\top} \delta$, but this means that the multi-beta model holds, although with 
parameters $\lambda+\delta$. Hence, we can conclude that $\alpha^{\star}=0$ is a necessary and sufficient condition for $\alpha=0$.

The next result obtains the relation between risk premia in the two formulations.

Result 2. We have

$$
\lambda^{\star}=\left(\gamma^{\star}\right)^{\top} \alpha+\Sigma_{y^{\star} y^{\star}} \Sigma_{y y}^{-1} \lambda
$$

where $\lambda^{\star}=\left(\gamma^{\star}\right)^{\top} E\left(r_{t}\right)$.

Hence, a sufficient condition for the risk premia in the two formulations to be the same is that $\alpha=0$, and that the factors are traded (in which case $\Sigma_{y^{\star} y^{\star}}=\Sigma_{y y}$ ).

\section{Mimicking portfolios}

\section{A. Cross-sectional regressions and mimicking portfolios}

The standard approach of running cross-sectional regressions (CSR) of average excess returns on betas leads to the risk-premium coefficients

$$
\tilde{\lambda}=\left(\beta W \beta^{\top}\right)^{-1} \beta W E\left(r_{t}\right)
$$

The CSR coefficients minimize the quadratic form

$$
\left[E\left(r_{t}\right)-\beta^{\top} \tilde{\lambda}\right]^{\top} W\left[E\left(r_{t}\right)-\beta^{\top} \tilde{\lambda}\right]
$$

Two common choices for the weighting matrix $W$ are $W=I$ and $W=\Sigma_{e e}^{-1}$. The first choice corresponds to an OLS regression; the second choice corresponds to a GLS regression. Alternatively we can choose $W=\Sigma_{r r}^{-1}$.

Interestingly, we obtain the same CSR coefficients for $W=\Sigma_{r r}^{-1}$ as for $W=\Sigma_{e e}^{-1}$. The intuition for this result is straightforward: minimizing the total portfolio variance with a constraint on systematic risk is equivalent to minimizing the idiosyncratic portfolio variance with a constraint on systematic risk. (Shanken, 1985, states, but does not prove, this result; in turn, he references his unpublished dissertation for a proof.) This result proves to be 
useful when we consider an alternative interpretation of the CSR coefficients. First, we can recognize that the $K \times N$ matrix

$$
\tilde{\gamma}^{\top}=\left(\beta W \beta^{\top}\right)^{-1} \beta W
$$

is a matrix of $K$ sets of $N$ portfolio weights. Each portfolio has unit beta w.r.t. the chosen factor and zero betas w.r.t. all other factors included in the LFM

$$
\tilde{\gamma}^{\top} \beta^{\top}=\left(\beta W \beta^{\top}\right)^{-1} \beta W \beta^{\top}=I
$$

On the other hand, it is straightforward to show how the CSR portfolio weights correspond to the solution of the minimization problem (see, for example, Litzenberger and Ramaswamy, 1979, and Huberman et al., 1987)

$$
\begin{aligned}
& \min _{\tilde{\gamma_{k}}}{\tilde{\gamma_{k}}}^{\top} W^{-1} \tilde{\gamma_{k}} \\
& \text { s.t. } \beta \gamma_{k}=s_{k},
\end{aligned}
$$

where $\tilde{\gamma}_{k}$ is the $N \times 1$ vector of portfolio weights; and $s_{k}$ is a vector with the $k$-th element equal to one, and zeros elsewhere.

Hence, when $W=I$, the CSR portfolio weights minimize the length of the vector of portfolio weights, subject to the unit-beta constraint. When $W=\Sigma_{r r}^{-1}$, the CSR portfolio weights minimize the variance of the mimicking portfolio returns, subject to the unit-beta constraint. This second set of portfolio weights is equal to the set of portfolio weights implicit in the CSR-GLS coefficients $\left(\beta \Sigma_{e e}^{-1} \beta^{\top}\right)^{-1} \beta \Sigma_{e e}^{-1} E\left(r_{t}\right)$.

Note that the maximum-correlation portfolios are proportional to the solution of the minimization of $\gamma_{k}^{\top} \Sigma_{r r} \gamma_{k}$ w.r.t. $\gamma_{k}$, subject to the single constraint $\Sigma_{y_{k} r} \gamma_{k}=1$. Hence, both unit-beta CSR-GLS portfolios and maximum-correlation portfolios minimize the variance of the portfolio returns subject to constraints on covariances. The difference is that the unitbeta portfolios are subject to constraints on covariances with all the other factors, whereas the maximum-correlation portfolios are only subject to a single constraint on the covariance with the factor being tracked.

One additional property of the unit-beta portfolio weights is that, for $W=\Sigma_{r r}^{-1}$ (or $\left.W=\Sigma_{e e}^{-1}\right)$, and in the case of a single factor, the weights are proportional to the maximum- 
correlation mimicking-portfolio weights. In fact

$$
\tilde{\gamma}^{\top}=\left(\beta \Sigma_{r r}^{-1} \beta^{\top}\right)^{-1} \beta \Sigma_{r r}^{-1}=\left(\Sigma_{y r} \Sigma_{r r}^{-1} \Sigma_{r y} / \sigma_{y}^{2}\right)^{-1} \Sigma_{y r} \Sigma_{r r}^{-1}
$$

Since $\left(\Sigma_{y r} \Sigma_{r r}^{-1} \Sigma_{r y} / \sigma_{y}^{2}\right)$ in the one-factor case is a scalar, $\tilde{\gamma}^{\top}$ above is proportional to $\Sigma_{y r} \Sigma_{r r}^{-1}=$ $\left(\gamma^{\star}\right)^{\top}$. In the general case of $K$ factors, on the other hand, the unit-beta mimicking-portfolio weights $\tilde{\gamma}$ are linear combinations of the maximum-correlation mimicking-portfolio weights $\gamma^{\star}$.

Note, though, that the discussion above does not mean that in the presence of a single non-traded factor, the properties of the estimates of the risk premium on the CSRGLS portfolio and on the maximum correlation portfolio are the same. In fact, while the maximum-correlation portfolios are constructed subject to a covariance constraint, the CSRGLS portfolios are constructed subject to a beta constraint. In general, covariance and beta estimates have different properties, and these leads to different properties of the risk-premium estimates. For example, in the case of a noisy factor (more on this in Section VI.B below), covariance estimates are consistent, while beta estimates are not. This leads to a different behavior of the two risk-premium estimates, even when there is only one factor at work.

Turning now to risk premia, the average excess returns on the unit-beta portfolios $\tilde{\lambda}$ coincide with the $\lambda$ parameters only under the null

$$
\tilde{\lambda}=\tilde{\gamma}^{\top} E\left(r_{t}\right)=\left(\beta W \beta^{\top}\right)^{-1} \beta W E\left(r_{t}\right)=\lambda+\left(\beta W \beta^{\top}\right)^{-1} \beta W \alpha
$$

In other words, under the alternative, the expected excess returns on the unit-beta portfolios do not have any direct relation with the $\lambda$ parameters. Moreover, the risk premia $\tilde{\lambda}$ depend on the choice of weighting matrix and are generally sensitive to the repackaging of securities.

Similarly, under the alternative, the alphas associated with the CSR premia are given by

$$
\tilde{\alpha}=E\left(r_{t}\right)-\beta^{\top}\left(\beta W \beta^{\top}\right)^{-1} \beta W E\left(r_{t}\right)=\left[I-\beta^{\top}\left(\beta W \beta^{\top}\right)^{-1} \beta W\right] \alpha .
$$

Hence, $\alpha=0$ implies $\tilde{\alpha}=0$, and $\alpha \neq 0$ implies $\tilde{\alpha} \neq 0$. On the other hand, $\tilde{\alpha}=0$ does not imply $\alpha=0$ since the $K \times K$ matrix $I-\beta^{\top}\left(\beta W \beta^{\top}\right)^{-1} \beta W$ is of rank $N-K$. As in Result 1 , though, the null space of the idempotent matrix $I-\beta^{\top}\left(\beta W \beta^{\top}\right)^{-1} \beta W$ is spanned by linear combinations of the columns of $\beta^{\top}$. ( These two statements can be proved using the same 
arguments used for the matrix $I-\left(\beta^{\star}\right)^{\top}\left(\gamma^{\star}\right)^{\top}$.) Hence, we can conclude that $\tilde{\alpha}=0$ is a necessary and sufficient condition for $\alpha=0$.

The next result shows how the alphas associated with the CSR premia equal the alphas of the $\mathrm{LFM}^{\star}$ representation when $W=\Sigma_{e e}^{-1}\left(\right.$ or $\left.W=\Sigma_{r r}^{-1}\right)$.

Result 3. When $W=\Sigma_{e e}^{-1}$, then $\alpha^{\star}=\tilde{\alpha}$.

\section{B. Mimicking portfolios and Sharpe ratios}

Consider the unit-beta mimicking portfolios. The maximum squared Sharpe ratio from investment in the unit-beta mimicking portfolios is given by

$$
E\left(r_{t}\right)^{\top} \tilde{\gamma}^{\top}\left(\tilde{\gamma}^{\top} \Sigma_{r r} \tilde{\gamma}\right)^{-1} \tilde{\gamma} E\left(r_{t}\right)=E\left(r_{t}\right)^{\top} W \beta^{\top}\left(\beta W \Sigma_{r r} W \beta^{\top}\right)^{-1} \beta W E\left(r_{t}\right)
$$

Using the decomposition $E\left(r_{t}\right)=\tilde{\alpha}+\beta^{\top} \tilde{\lambda}$, where $\beta W \tilde{\alpha}=0$ by construction $(\tilde{\alpha}$ is the vector of residuals of a weighted least squares regression of the expected excess returns on the betas), the maximum squared Sharpe ratio simplifies to

$$
\tilde{\lambda}^{\top}(\beta W \beta)\left(\beta W \Sigma_{r r} W \beta^{\top}\right)^{-1}\left(\beta W \beta^{\top}\right) \tilde{\lambda}
$$

Note that $\left(\beta \Sigma_{e e}^{-1} \beta^{\top}\right)^{-1} \beta \Sigma_{e e}^{-1}=\left(\beta \Sigma_{r r}^{-1} \beta^{\top}\right)^{-1} \beta \Sigma_{r r}^{-1}$ and the unit-beta portfolio weights are the same for the two choices of weighting matrix $W=\Sigma_{e e}^{-1}$ and $W=\Sigma_{r r}^{-1}$. Substituting $W=\Sigma_{r r}^{-1}$ in the expression above, we obtain

$$
\tilde{\lambda}^{\top} \beta \Sigma_{r r}^{-1} \beta^{\top} \tilde{\lambda}
$$

Hence, in the special case where $W=\Sigma_{e e}^{-1}$, if the multi-beta result holds $(\lambda=\tilde{\lambda}$ and $\left.\beta^{\top} \tilde{\lambda}=E\left(r_{t}\right)\right)$ then the maximum squared Sharpe ratio attainable from investment in the unit-beta portfolios equals the maximum squared Sharpe ratio attainable from investment in all of the assets available.

Consider now the maximum-correlation mimicking portfolios. The maximum squared Sharpe ratio from investing in the maximum-correlation mimicking portfolios is

$$
E\left(y_{t}^{\star}\right)^{\top} \Sigma_{y^{\star} y^{\star}}^{-1} E\left(y_{t}^{\star}\right)=E\left(r_{t}\right)^{\top} \gamma^{\star}\left(\left(\gamma^{\star}\right)^{\top} \Sigma_{r r} \gamma^{\star}\right)^{-1}\left(\gamma^{\star}\right)^{\top} E\left(r_{t}\right)
$$


Using again the decomposition $E\left(r_{t}\right)=\tilde{\alpha}+\beta^{\top} \tilde{\lambda}$ and the fact that $\Sigma_{y r} W \tilde{\alpha}=0(\tilde{\alpha} \equiv$ $\left.E\left(r_{t}\right)-\beta^{\top} \tilde{\lambda}=E\left(r_{t}\right)-\Sigma_{r y}\left(\Sigma_{y r} W \Sigma_{r y}\right)^{-1} \Sigma_{y r} W E\left(r_{t}\right)=\left[I-\Sigma_{r y}\left(\Sigma_{y r} W \Sigma_{r y}\right)^{-1} \Sigma_{y r} W\right] E\left(r_{t}\right)\right)$, we have

$$
E\left(y_{t}^{\star}\right)^{\top} \Sigma_{y^{\star} y^{\star}}^{-1} E\left(y_{t}^{\star}\right)=\tilde{\lambda}^{\top} \beta \Sigma_{r r}^{-1} \beta^{\top} \tilde{\lambda}
$$

which, not surprisingly, is the same as the maximum squared Sharpe ratio from investing in the unit-beta mimicking portfolios, for $W=\Sigma_{e e}^{-1}$ This means that, in the case of a single factor, the Sharpe ratios on the two types of mimicking portfolios are the same. Again, under the null, the investor is able to achieve the same Sharpe ratio from investing in the maximum-correlation mimicking portfolios as from investing in the original assets.

\section{Moment conditions}

In the following, we illustrate the moment conditions imposed in the estimation of the two formulations of the linear factor model.

\section{A. LFM}

Let $z_{t}$ denote the data and $\theta$ the parameter vector. The moment conditions $E\left[f\left(z_{t}, \theta\right)\right]=0$ for the LFM representation are given by

$$
\begin{aligned}
E\left(r_{t}-\beta_{0}-\beta^{\top} y_{t}\right) & =0 \\
E\left[\left(r_{t}-\beta_{0}-\beta^{\top} y_{t}\right) \otimes y_{t}\right] & =0 \\
E\left(r_{t}-\beta^{\top} \lambda\right) & =0 .
\end{aligned}
$$

Following Hansen (1982), we set a linear combination of the moment conditions equal to zero, i.e. $a \hat{E}\left[f\left(z_{t}, \hat{\theta}\right)\right]=0$, where

$$
a=\left[\begin{array}{cc}
I_{N(K+1)} & 0_{N(K+1), N} \\
0_{K, N(K+1)} & \hat{\beta} W
\end{array}\right] .
$$

We consider two weighting matrices: $W=I$ and $W=\hat{\Sigma}_{e e}^{-1}$. 
The resulting $\lambda$ estimates are given by

$$
\hat{\lambda}=\left(\hat{\beta} W \hat{\beta}^{\top}\right)^{-1} \hat{\beta} W \hat{E}\left(r_{t}\right) .
$$

For $W=\hat{\Sigma}_{e e}^{-1}\left(\right.$ or $\left.W=\hat{\Sigma}_{r r}^{-1}\right)$, the resulting $\lambda$ estimates coincide with those of the optimal GMM estimator (in the i.i.d. case),

$$
a=\left.\frac{\partial \hat{E}\left[f\left(z_{t}, \theta\right)\right]^{\top}}{\partial \theta}\right|_{\theta=\hat{\theta}} \hat{\Sigma}_{f f}^{-1},
$$

conditional on $\hat{\beta}$.

Relative to the approach of using the optimal weighting matrix as in Jagannathan and Wang (2002) and Kan and Zhou (1999, 2001a), we see two main advantages to our approach. First, all estimates are obtained in closed-form, which is especially useful in the simulation exercise. Second, the estimates of the risk premium parameters $\lambda$ are the same as those obtained with the traditional CSR approach.

\section{B. LFM $^{\star}$}

In the $\mathrm{LFM}^{\star}$ formulation, the moment conditions are given by

$$
\begin{aligned}
E\left[y_{t}-\gamma_{0}^{\star}-\left(\gamma^{\star}\right)^{\top} r_{t}\right] & =0 \\
E\left\{\left[y_{t}-\gamma_{0}^{\star}-\left(\gamma^{\star}\right)^{\top} r_{t}\right] \otimes r_{t}\right\} & =0 \\
E\left[\left(\gamma^{\star}\right)^{\top} r_{t}-\lambda^{\star}\right] & =0 \\
E\left[r_{t}-\alpha^{\star}-\left(\beta^{\star}\right)^{\top} y_{t}^{\star}\right] & =0 \\
E\left\{\left[r_{t}-\alpha^{\star}-\left(\beta^{\star}\right)^{\top} y_{t}^{\star}\right] \otimes y_{t}^{\star}\right\} & =0 .
\end{aligned}
$$

In this case, the estimates are obtained by exactly-identified GMM $(a=I)$.

One potential disadvantage of this second approach is that we now have a total of $K(N+$ $1)+K+N(K+1)$ parameters to estimate, to be compared with $K+N(K+1)$ parameters for the LFM representation. Hence, one concern in the simulation analysis will be to see if the larger number of parameters to estimate affects the small-sample properties of the estimates. 


\section{Asymptotics}

For both representations, the asymptotic covariance matrix of the estimates is given by

$$
\operatorname{Cov}(\hat{\theta})=\frac{1}{T}(a d)^{-1} a S a^{\top}\left[(a d)^{-1}\right]^{\top},
$$

where

$$
d=\left.\frac{\partial \hat{E}\left[f\left(z_{t}, \theta\right)\right]}{\partial \theta^{\prime}}\right|_{\theta=\hat{\theta}},
$$

and

$$
S=\sum_{j=-\infty}^{\infty} \hat{E}\left[f\left(z_{t}, \hat{\theta}\right) f\left(z_{t-j}, \hat{\theta}\right)^{\top}\right] .
$$

It is worth noting that for the $\mathrm{LFM}^{\star}$ representation, the matrix $S$ does not have full rank $K+K N+K+N+N K$, but instead has rank equal to $(K+K N+K+N+N K)-K-K^{2}$ The reason for this is that one can construct $K$ linear combinations of the excess returns that exactly replicate the excess returns on the maximum-correlation mimicking portfolios. Hence, for $K$ linear combinations of the excess returns, the residuals of the regression on the excess returns on the maximum-correlation mimicking portfolios are identically zero. This also means that the $K^{2}$ products of these residuals with the excess returns on the maximum-correlation mimicking portfolios are zero. One way to get around this problem would be to differentiate the set of the test assets from the set of assets used to construct the mimicking portfolios. Indeed, this is the approach used in the maximum-likelihood estimation of Breeden, Gibbons, and Litzenberger (1989). Note, though, that in our GMM setting the singularity of $S$ does not present a problem in estimation, since it does not need to be inverted. Moreover, the inversion of portions of the covariance matrix of the estimates is indeed possible. For example, we can invert the covariance matrix of the $\alpha^{\star}$ estimates for the purpose of computing Wald-style statistics. Note that a non-invertible $S$ matrix is not unique to our setting. Kan and Zhou (2001b) also obtain a non-invertible $S$ matrix in the case of exactly-identified GMM spanning tests using the stochastic-discount-factor approach. Peñaranda and Sentana (2004), on the other hand, consider the case of over-identified GMM spanning tests, when $S$ is not invertible. 
The covariance matrix of the estimates is used to test the joint significance of the $\alpha^{\star}$ estimates in the $\mathrm{LFM}^{\star}$ representation. In the LFM representation, on the other hand, the $\alpha$ estimates are the averages of the last set of $K$ moment conditions. Hence, the joint significance of the $\alpha$ estimates is tested based on the covariance matrix

$$
\operatorname{Cov}\left\{\hat{E}\left[f\left(z_{t}, \hat{\theta}\right)\right]\right\}=\frac{1}{T}\left[I-d(a d)^{-1} a\right] S\left[I-d(a d)^{-1} a\right]^{\top} .
$$

\section{Special cases}

\section{A. Traded factors}

When a subset $y_{1 t}$ of the factors $y_{t}$ are traded, the analytical results of the previous sections, as well as the estimation approach, are slightly changed. This is because we introduce the restriction that the risk premium-estimates for the traded factors are simply the time-series averages of the traded factors (see, for example, Shanken, 1992). Let

$$
r_{2 t} \equiv r_{t}-\beta_{1}^{\top} y_{1 t}
$$

where $\beta_{1}$ are the coefficients of a projection of $r_{t}$ onto the span of $y_{1 t}$. ( It is worth noting that the analysis that follows would go through for slightly different definitions of $r_{2 t}$ : $\beta_{1}$ could be the coefficients of a projection including an intercept, or $\beta_{1}$ could be a subset of the coefficients of a projection of $r_{t}$ on both $y_{1 t}$ and $y_{2 t}$.) Also, let $\beta_{2}$ denote the coefficients of the projection of $r_{2 t}$ onto the augmented span of the non-traded factors; and let $\gamma_{2}^{\star}$ denote the coefficients of the projection of the non-traded factors onto the augmented span of $r_{2 t}$.

The LFM representation leads to the mimicking-portfolio coefficients

$$
\tilde{\gamma}_{2}^{\top}=\left(\beta_{2} W \beta_{2}^{\top}\right)^{-1} \beta_{2} W
$$

and the risk premia

$$
\tilde{\lambda}_{2}=\left(\beta_{2} W \beta_{2}^{\top}\right)^{-1} \beta_{2} W E\left(r_{2 t}\right)
$$

The $\mathrm{LFM}^{\star}$ representation leads to the risk premia

$$
\lambda_{2}^{\star}=\left(\gamma_{2}^{\star}\right)^{\top} E\left(r_{2 t}\right)
$$


Hence, all the analytical results of the previous sections still hold, but where $r_{t}$ is replaced by $r_{2 t} ; \beta$ is replaced by $\beta_{2} ; y_{t}$ is replaced by $y_{2 t}$; and $\gamma^{\star}$ is replaced by $\gamma_{2}^{\star}$.

For estimation, the same modifications take place. For the LFM specification, we have the orthogonality conditions

$$
\begin{aligned}
E\left(r_{t}-\beta_{1}^{\top} y_{1 t}\right) & =0 \\
E\left(r_{2 t}-\beta_{0}-\beta_{2}^{\top} y_{2 t}\right) & =0 \\
E\left[\left(r_{2 t}-\beta_{0}-\beta_{2}^{\top} y_{2 t}\right) \otimes y_{2 t}\right] & =0 \\
E\left(r_{2 t}-\beta_{2}^{\top} \lambda_{2}\right) & =0 .
\end{aligned}
$$

In constructing the a matrix, $\hat{\beta}_{2}$ replaces $\hat{\beta}$, while $W$ can either equal $I$ or $\hat{\Sigma}_{e e}^{-1}$. For the $\mathrm{LFM}^{\star}$ formulation, we have the orthogonality conditions

$$
\begin{aligned}
E\left(r_{t}-\beta_{1}^{\top} y_{1 t}\right) & =0 \\
E\left[y_{2 t}-\gamma_{0}^{\star}-\left(\gamma_{2}^{\star}\right)^{\top} r_{2 t}\right] & =0 \\
E\left\{\left[y_{2 t}-\gamma_{0}^{\star}-\left(\gamma_{2}^{\star}\right)^{\top} r_{2 t}\right] \otimes r_{2 t}\right\} & =0 \\
E\left(y_{2 t}^{\star}-\mu_{y_{2}^{\star}}\right) & =0 \\
E\left[r_{2 t}-\alpha^{\star}-\left(\beta_{2}^{\star}\right)^{\top} y_{2 t}^{\star}\right] & =0 \\
E\left\{\left[r_{t}-\alpha^{\star}-\left(\beta_{2}^{\star}\right)^{\top} y_{2 t}^{\star}\right] \otimes y_{2 t}^{\star}\right\} & =0 .
\end{aligned}
$$

\section{B. Noisy factors}

It is straightforward to show that in the case of a single noisy factor, the CSR-OLS estimator leads to inconsistent estimates of the risk premium $\lambda$ ( see Kan and Zhang, 1999, for a similar discussion). Assume $\hat{y}_{t}=y_{t}+\epsilon_{t}$, where $E\left(y_{t} \epsilon_{t}\right)=0$ and $E\left(r_{t} \epsilon_{t}\right)=0$.

We have

$$
\operatorname{Plim} \hat{\beta}=\frac{\operatorname{Var}\left(y_{t}\right)}{\operatorname{Var}\left(\hat{y}_{t}\right)} \beta
$$

Consider the CSR $\lambda$ estimator for $W=I$. We have

$$
\operatorname{Plim} \hat{\lambda}=\frac{\operatorname{Var}\left(\hat{y}_{t}\right)}{\operatorname{Var}\left(y_{t}\right)} \lambda
$$


In other words, the attenuation bias in the $\beta$ estimates translates into an upward bias in the $\lambda$ estimates. On the other hand, it is immediate to show that the $\gamma^{\star}$ and $\lambda^{\star}$ estimates are still consistent.

\section{Data}

We calibrate the simulation experiment using monthly data from 1959:03 to 2002:11 (525 obs.). We consider two sets of test assets: ten size-sorted portfolios (value-weighted (VW) NYSE-AMEX-NASDAQ decile portfolios, from CRSP); and the 25 Fama-French portfolios (from French's web page). We consider the following three factors: log real per-capita consumption growth (nondurable goods and services, CITIBASE); the VW NYSE-AMEXNASDAQ index return in excess of the risk-free rate (CRSP); and the change in the dividend yield on the composite S\&P500, (CITIBASE). As a proxy for the risk-free rate, we use the nominal one-month Treasury bill rate from CRSP.

\section{Bootstrap experiment}

In the following, we provide a detailed description of the bootstrap experiment

\section{Return generating process}

We simulate a one-factor C-CAPM economy and a two-factor I-CAPM economy. In the one-factor economy, the factor is the change in the log of real per-capita consumption, $y_{c t}$

$$
r_{t}=\alpha+\beta_{c}^{\top}\left[y_{c t}-E\left(y_{c t}\right)+\lambda_{c}\right]+e_{t} .
$$

In the two-factor economy, the traded factor is the excess return on the market, $y_{m t}$, and the non-traded factor is the change in the dividend yield, $y_{d t}$

$$
r_{t}=\alpha+\beta_{m}^{\top} y_{m t}+\beta_{d}^{\top}\left[y_{d t}-E\left(y_{d t}\right)+\lambda_{d}\right]+e_{t}
$$

\section{Calibration}


Betas, alphas, and risk premia for the LFM are estimated using the approach described in Section IV, where we set $W=I$. For both economies, we consider two choices of reference parameters, corresponding to two choices of assets: the ten size portfolios and the 25 Fama-French portfolios.

\section{Bootstrap}

We jointly bootstrap the realizations of the factors and the realizations of the residuals of the LFM. This means that we are sampling from a distribution where the factors and the residuals are orthogonal, but where they may display higher-order dependence. Since we are drawing from the empirical distribution of factors and residuals, we are not imposing distributional assumptions; in particular, we are not imposing normality. We set $\alpha=0$ in all simulations, with the exception of the analysis of power, where $\alpha$ is set to its estimated value. We consider two lengths of the data set: the full sample of 525 observations and a shorter sample of 240 observations (the first 240 observations in our sample). We implement both an i.i.d. and a block bootstrap (blocks of three monthly observations; see Cochrane, 2001).

\section{Empirical analysis of simulated data}

For each string of simulated data, we estimate the parameters of the LFM based on the two weighting matrices $W=I$ and $W=\Sigma_{e e}^{-1}$. Estimates of the parameters of the $\mathrm{LFM}^{\star}$ are obtained by exactly-identified GMM, and do not require a choice of weighting matrix. Asymptotic statistics for each simulation are obtained assuming no serial correlation (for the i.i.d. bootstrap), or serial correlation of order three (for the block bootstrap), with Newey and West (1987) adjustment. 


\section{Results}

\section{A. Estimates of economic risk premia}

\section{A.1. C-CAPM}

Table II reports results for the one-factor (C-CAPM) case. Panels A and B report statistics for the $\lambda$ estimates, while Panel $\mathrm{C}$ reports results for the $\lambda^{\star}$ estimates.

The table presents population values for the risk premia, average risk-premium estimates, biases, and RMSEs. For ease of comparison, we report risk premia as percentages of the population standard deviation of the corresponding mimicking-portfolio excess return. Given the results of Section III, this means that the reference values for the CSR-GLS estimates and the $\lambda^{\star}$ estimates are the same; whereas the reference values for CSR-OLS estimates and CSR-GLS $/ \lambda^{\star}$ estimates differ. This is because the same population risk premium is divided by different population values of the standard deviations of the mimicking-portfolio excess returns. Biases and RMSEs are reported as percentages of the population standard deviation of the corresponding mimicking-portfolio excess return, and also as percentages of the population absolute values of the risk premia (in parenthesis).

The table also reports averages and standard deviations of asymptotic t-ratios on the riskpremium estimates. We compute t-ratios using the population risk premium as the reference value. Finally, the table reports the ratios between the average asymptotic standard error on the risk-premium estimate and the standard deviation of the estimate across simulations.

\section{LFM}

Consider first the results for the estimates of $\lambda$ (Table II, Panel A and B). OLS estimates are biased away from zero, with biases ranging between 0.27 and 1.51, and with percentage biases ranging between $2.30 \%$ and $13.05 \%$. On the other hand, GLS estimates are biased towards zero, with biases ranging between -10.62 and -2.88 , and with percentage biases ranging between $-59.08 \%$ and $-18.16 \%$. In all instances, the absolute percentage bias is larger for the GLS estimates than for the OLS estimates. RMSEs are substantial for both estimators. They range between 5.48 and 11.07 (between $46.72 \%$ and $95.68 \%$ of the true value) for the OLS estimator, and between 5.28 
and 11.73 (between $33.29 \%$ and $65.31 \%$ of the true value) for the GLS estimator. Percentage RMSEs are always larger for the OLS than for the GLS estimator. As one would expect, reducing the length of the sample increases biases and RMSEs. Using the Fama-French, rather than the size portfolios, leads to smaller (larger) biases and RMSEs for the OLS (GLS) estimates. Going from the i.i.d. bootstrap of Panel A to the block bootstrap of Panel B makes little difference.

Asymptotic t-ratios have means that are substantially different from 0, ranging between -2.39 and -0.12 ; the biases are always more pronounced for the GLS than for the OLS estimator. The standard deviations of the asymptotic t-ratios also can deviate substantially from the theoretical value of one, ranging between 0.93 and 1.25. The deviations are always more pronounced for the GLS than for the OLS estimator. Finally, the ratios between asymptotic and empirical standard deviations are mainly lower than one for both choices of weighting matrix, ranging between 0.94 and 1.01, without a clear superiority of one estimator or the other.

\section{LFM* $^{*}$}

Results for the estimates of $\lambda^{\star}$ (Table II, Panel C) can be directly compared with the results for the CSR-GLS estimates, since the population Sharpe ratios for the two mimicking portfolios are the same. There is an advantage in using the $\mathrm{LFM}^{\star}$ formulation in terms of bias: absolute biases of the $\lambda^{\star}$ estimates are always substantially smaller than the biases of the corresponding CSR-GLS estimates. For example, in the i.i.d. bootstrap, 240 months, and Fama-French portfolios, the bias for the CSR-GLS estimator is -10.62 , whereas it is only -1.95 for the $\lambda^{\star}$ estimator. Notice that, under normality, the estimates of $\lambda^{\star}$ would be unbiased (Dickey, 1967). Hence, deviations from normality (e.g. skewness and excess kurtosis) in our bootstrap data are responsible for the biases that we document. RMSEs are roughly of the same magnitude as those for the GLS estimates.

Percentage biases for the $\lambda^{\star}$ estimator range between $-10.97 \%$ and $-0.69 \%$, and are higher or lower than the percentage biases for the CSR-OLS estimates depending on the choice of test assets. Percentage RMSEs for the $\lambda^{\star}$ estimator range between $35.52 \%$ 
and $63.75 \%$, again higher or lower than the corresponding quantities for the CSR-OLS estimates depending on the choice of test assets. Hence, the performance of $\lambda^{\star}$ and CSR-OLS estimates in terms of bias and RMSE is roughly comparable.

T-ratios in the $\mathrm{LFM}^{*}$ are negatively biased: biases are similar to the OLS estimates and much less pronounced than for the GLS estimates. The standard deviations of the t-ratios are consistently less than one, also similar to the OLS estimates. Finally, the ratios between asymptotic standard errors and empirical standard errors are consistently larger than one, differently from the OLS and GLS estimates.

\section{A.2. I-CAPM}

Table III reports the same results for the multi-factor (I-CAPM) case. Panels A and B report results for the $\lambda$ estimates, while Panel $\mathrm{C}$ reports results for the $\lambda^{\star}$ estimates. In this case, we focus on the risk premia on the non-traded factor: changes in the dividend yield.

\section{LFM}

As in the one-factor case, the GLS estimates are biased towards zero (the bias is positive, while the true value of the parameter is negative); on the other hand, the bias in the OLS estimates changes sign depending on the set of assets. Again as in the one-factor case, percentage biases are more pronounced for the GLS estimates, ranging between $23.39 \%$ and $51.54 \%$; while biases range between $-9.85 \%$ and $7.26 \%$ for the OLS estimates. Percentage RMSEs are substantial for both sets of estimates and of roughly similar magnitude, ranging between $38.53 \%$ and $116.42 \%$. Consistent with the one-factor case, RMSEs are always larger for the OLS than the GLS estimates.

Biases in t-ratios are now consistently positive for both estimators; as in the one-factor case, they are more pronounced for the GLS estimator. As in the one-factor case, standard deviations of t-ratios are below one for the OLS estimator and above one

for the GLS estimator. Finally, the ratios between asymptotic and empirical standard errors of the estimates are mainly below one, also consistent with the one-factor case.

\section{LFM* $^{*}$}


As in the one-factor case, $\lambda^{\star}$ estimates are biased towards zero; hence, biases are now positive. Again, biases are consistently smaller than for the corresponding GLS estimates. For example, in the i.i.d. bootstrap, 240 months, and Fama-French portfolios, the bias for the CSR-GLS estimator is 8.64, whereas it is only 2.93 for the $\lambda^{\star}$ estimator (the reference value is -16.92). RMSEs are again comparable to those for the GLS estimates.

Comparing $\lambda^{\star}$ estimates to CSR-OLS estimates, we now find that percentage biases are always more pronounced for the $\lambda^{\star}$ estimate. Percentage RMSEs, on the other hand, are similar for the two estimators, with their relative size depending on the choice of test assets, just as in the one-factor case.

Biases in t-ratios are positive, less pronounced than for the CSR-OLS estimator and comparable to the CSR-GLS estimator, as in the one-factor case. The volatility of t-ratios is now consistently higher than one, and, in comparing departures from the true value of one, no one estimator clearly outperforms the other two. Finally, the ratios between asymptotic and empirical standard errors of the estimates are always higher than one, and tend to depart from the reference value of one more than the other two estimators, consistent with the one-factor case.

\section{A.3. Summary and discussion}

In summary, the various scenarios considered paint a fairly consistent picture of the advantages and disadvantages of the various estimators. In terms of bias, the $\lambda^{\star}$ estimates always do better than the CSR-GLS estimates, while they often do worse than the CSR-OLS estimates. In terms of RMSE, the $\lambda^{\star}$ estimates always do better than the CSR-OLS estimates, while they perform similarly to the CSR-GLS estimates. In terms of average t-ratios, the $\lambda^{\star}$ estimates always do better than the CSR-GLS estimates, and they perform similarly to the CSR-OLS estimates.

We can compare the results above to the results obtained by Chen and Kan (2006), in a similar setting. Chen and Kan derive analytically the small-sample bias and standard deviation of CSR estimates for the OLS and GLS cases, under the assumption of i.i.d. 
Gaussian returns and factor; and they perform a simulation exercise for non-normal (studentt) returns and factor. They consider the case where the factor is calibrated to consumption growth, and they consider both size-sorted and size- and value-sorted test portfolios. Several of their results are consistent with ours. They find that absolute biases are larger for the GLS than for the OLS estimates, that the GLS estimates are consistently biased towards zero, and that the sign of the bias for the OLS estimates can be either positive or negative, depending on the calibration. They find that the GLS estimates are less volatile than the OLS estimates, which is consistent with our finding that the RMSEs are smaller for the GLS estimates.

\section{B. Changing betas}

In this section, we further investigate the nature of the difference in results between the LFM and $\mathrm{LFM}^{\star}$ formulations. We replicate the one-factor case, for the scenario with i.i.d. bootstrap, full sample, and size portfolios, but where the betas w.r.t. consumption growth are scaled by a factor $m$. The variances of the idiosyncratic components are adjusted so that the variances of returns remain the same. Hence, as betas increase in absolute value, the percentages of return variance explained by the single-index model also increase.

Results are reported in Table IV. The table clearly shows how the biases in the $\lambda$ estimates tend to zero as the consumption betas increase from $1 / 3$ to 3 times the original value. This pattern is especially pronounced for the CSR-GLS estimate, whose bias goes from 11.22 to -0.43 (from $-68.62 \%$ to $-3.36 \%$ ). On the other hand, the bias in the $\lambda^{\star}$ estimates remains remarkably stable (between -0.32 and $-0.14 ;-1.96 \%$ and $-1.10 \%$ of the population values, respectively) across values of $m$. As in the scenarios illustrated in the previous section, the percentage RMSEs of the CSR-GLS and $\lambda^{\star}$ estimates are comparable, while percentage RMSEs of the CSR-OLS estimator are consistently higher. RMSEs also decrease monotonically as the consumption betas increase (in absolute value) although they are still substantial for $m=3$, ranging between $34.04 \%$ and $44.56 \%$.

In summary, we conclude that the discrepancies in results across the different riskpremium estimators tend to disappear as the factor explains more of the variance of returns. 


\section{Noisy factor}

Table $\mathrm{V}$ shows results for the one-factor case, when the factor is observed with noise. We focus on one scenario only: i.i.d. bootstrap, full sample, and size portfolios. Following Kan and Zhou (1999), we consider noise with standard deviation equal to 0.5, 1, 1.5, and 2 times the standard deviation of the factor.

A setting where the issue of noise in measuring the factor arises naturally is that of tests of heterogenous-agent models; see, for example, Brav, Constantinides, and Geczy (2002), and Jacobs and Wang (2004). In these studies, the realizations of the factors are the moments of the cross-sectional distribution of consumption growth. Since the cross-sections used in these studies are relatively small, the cross-sectional moments can be estimated with a great deal of noise.

The table presents reference values of the risk premia, biases, RMSEs, and ratios between average asymptotic standard errors and empirical standard deviations of the estimates. In this setting, the results are dramatically in favor of the LFM ${ }^{\star}$ formulation over both the CSR-OLS and CSR-GLS approaches. Bias and RMSE monotonically increase with noise for the CSR $\lambda$ estimates. For example, when noise is twice as large as the signal, the biases for the CSR-OLS and CSR-GLS estimates are 48.95 and 18.54 (423.08\% and 116.90\%), respectively; and RMSEs are as high as 79.97 and 26.28 (691.18\% and $165.70 \%)$. On the other hand, the bias for the $\lambda^{\star}$ estimates never exceeds $0.13(0.82 \%)$ in absolute value, with a maximum RMSE of 9.10 (57.38\%).

\section{Estimates of betas}

We analyze the finite sample properties of $\beta$ and $\beta^{\star}$. Note that the $\beta^{\star}$ estimates are subject to the EIV problem arising from the estimation of the weights of the maximum-correlation mimicking portfolio. We conduct simulations to assess the magnitude of this bias.

Table VI reports results for the C-CAPM case. We report the same statistics as in Table II, averaged across assets. For ease of comparison, both $\beta$ and $\beta^{\star}$ estimates are scaled by the ratio between the population standard deviations of the factor and of the dependent variable. Hence, they have the dimension of correlation coefficients. Percentage biases and 
RMSEs are computed as average biases and RMSEs across assets divided by the population values of the betas, averaged across assets.

Panel A shows results for the estimates of $\beta$. Biases are essentially non-existent, although RMSEs are substantial (as high as 38.48\%). Similarly unbiased are, on average, the t-ratios. The volatilities of the t-ratios are close to one, and the ratios between average asymptotic standard errors and empirical standard errors are also close to one.

Panel B shows results for the estimates of $\beta^{\star}$. Biases are negative (this is the well known "attenuation bias" associated with the EIV problem) and substantial, ranging between -29.67 and $-11.84(-53.30 \%$ and $-17.04 \%)$. RMSEs are also substantial, as high as $31.63(56.82 \%)$, although of the same oder of magnitude as for the LFM specification. Finally, biases in t-ratios are negative and pronounced, and there are substantial discrepancies between small sample and asymptotic volatilities of t-ratios.

Table VII reports results for the I-CAPM. In this case, we focus on the betas for the non-traded factor. As in the single-factor case, biases and RMSEs are more pronounced for the $\beta^{\star}$ estimates than for the $\beta$ estimates.

\section{E. Size and power of the tests}

We investigate by simulation the size and power properties of the Wald-style tests for the one-factor and two-factor models. The main contribution consists in the analysis of the size and power properties of tests in the context of the LFM^ representation.

\section{E.1. Size}

Table VIII reports the theoretical and actual sizes of the Wald-style test for the one-factor model. Panels A and B report results for the LFM specification, for the i.i.d. and the block bootstrap, respectively. Panel C reports results for the $\mathrm{LFM}^{\star}$ specification.

Consider first results for the LFM specification, full sample, i.i.d. bootstrap. The actual size is generally close to the theoretical value, although there are systematic discrepancies. The CSR-OLS approach always leads to lower rejection rates than the CSR-GLS approach; and using the Fama-French portfolios leads to higher rejection rates than using the size- 
sorted portfolios. Results are similar for the block bootstrap and for the shorter sample of 240 months. Indeed, rejection rates are quite similar for the two sample lengths.

When we consider the $\mathrm{LFM}^{\star}$ specification, overall we see over-rejections. Again, rejection rates are stronger for the Fama-French portfolios. Rejection rates for the LFM* specification are always higher than rejection rates for the LFM specification, both OLS and GLS approaches.

Table IX, organized in the same way as Table VIII, presents results for the I-CAPM. The same patterns arise as in the one-factor specification.

\section{E.2. Power}

Tables X and XI (organized similarly to Tables VIII and IX) reports rejection rates for the Wald test under the alternative, where the size is adjusted using the bootstrap results of Table VIII: we compute the $10 \%, 5 \%$, and $1 \%$ quantiles of the empirical distribution of the Wald statistic under the null, and we compute the percentage of times the Wald statistic exceeds the corresponding quantile, when the economy is simulated under the alternative.

Consider first the case of the C-CAPM (Table X).

In the case of the LFM specification, the power of the tests is higher for $W=\hat{\Sigma}_{e e}^{-1}$, for the Fama-French portfolio returns, and for the longer sample. For example, consider the case of the i.i.d. bootstrap for the full sample and size-sorted portfolios. When the size is set at 1\%, the actual rejection rate is $57 \%$ for the OLS approach; the rejection rate increases to $80 \%$ for the GLS approach; the rejection rate further increases to $98 \%$ for the choice of Fama-French test portfolios; and the rejection rate decreases to $15 \%$ when the sample is shortened to 240 months.

In the case of the LFM $^{\star}$ specification, the power is also higher for the Fama-French portfolio returns and for the longer sample. For example, in the case of the i.i.d. bootstrap for the entire sample, size portfolios, when the size is set at $1 \%$, the rejection rate is $78 \%$; the rejection rate increases to $100 \%$ for the Fama-French portfolios; the rejection rate falls to $22 \%$ for the shorter sample. Power for the LFM ${ }^{\star}$ specification is always higher than power for the LFM specification, OLS approach, and comparable to power for the GLS approach.

For the I-CAPM (Table XI, organized as Table X) results are similar. In the case of the 
LFM specification, the power of the tests is almost always higher for $W=\Sigma_{e e}^{-1}$, although the differences are modest. Power is also higher for tests using the Fama-French portfolios and for the longer sample. Power for the $\mathrm{LFM}^{\star}$ specification is comparable to power for the LFM specification, GLS approach, and always higher than power than for the LFM specification, OLS approach.

Hence, when it comes to power, the CSR-GLS and LFM^ approaches have an advantage over the CSR-OLS approach, especially in the one-factor case. Also, it is a clear advantage to use the larger cross-section of the Fama-French portfolios, in both the one-factor and the two-factor case.

\section{Extensions}

In this section we consider several extensions of our main analysis. First, we consider the case of time-varying conditional moments and conditional versions of the LFM and LFM* formulations. Second, we consider an alternative approach to the construction of mimicking portfolios, first suggested by Lehmann and Modest (1988). Third, we consider the performance of the correction for small-sample bias in risk-premium estimates, suggested by Chen and Kan (2006). Fourth, we study the performance of the various methods when we consider long-horizon overlapping returns. Finally, we consider the implications of the restrictions from the LFM and $\mathrm{LFM}^{\star}$ formulations on covariance matrices, for the purpose of portfolio construction.

Details of the various exercises are in a separate appendix available from the authors upon request. In the following, we provide summaries of the various set-ups and results.

\section{A. Conditional models}

We consider conditional versions of the LFM and LFM^ formulations. We allow for both risk premia and betas to be time varying, and we also specialize the analysis to the cases where betas or risk premia are constant. Following Ferson and Harvey (1999), we assume that the conditional betas and the conditional expectations of the factors are linear in the instruments. Moreover, following Ferson and Harvey (1991), we assume that the conditional 
risk premia are also linear in the instruments. For tractability, we assume that while the betas are time-varying, the factors are homoskedastic (the same assumption is made, for example, in the simulation analysis of Ferson, Siegel, and $\mathrm{Xu}, 2005)$. We focus on conditional versions of the I-CAPM, where innovations in the dividend yield are the non-traded factor, and lagged realizations of the dividend yield drive the time-varying betas and dividend-yield risk premium. Conditional betas with respect to the traded factor, the market, can also vary as a function of the dividend yield.

Relative to the unconditional case, the properties of all estimators worsen considerably, when betas are allowed to vary. For example, the percentage bias of the CSR-OLS estimate increases from $-3.69 \%$ to $79.88 \%$, and the percentage RMSE increases from $67.44 \%$ to 193.91\%. When betas are kept constant, on the other hand, results are comparable to the base case illustrated in Table II. In terms of the relative performance of the three estimators, the CSR-OLS estimator still displays the lowest bias, but the highest RMSE, across scenarios. The $\lambda^{\star}$ estimate displays the lowest percentage RMSE when betas are time-varying. The CSR-GLS estimator has the lowest percentage RMSE when betas are constant.

Interestingly, while the properties of the estimates worsen, the correlations between mimicking-portfolio returns and the factor can increase substantially. For example, in the case where betas and risk premia are both time-varying, the correlations for the conditional mimicking portfolios are: $18.76 \%$ (CSR-OLS), 26.83\% (CSR-GLS), and 26.50\% (maximumcorrelation). For a comparison, the correlations for the unconditional mimicking portfolios are $16.69 \%, 20.76 \%$, and $20.76 \%$.

\section{B. Lehmann and Modest (1988) portfolios}

Lehmann and Modest (LM, 1988) argue that constructing unit-beta mimicking portfolios tends to place large weights on security returns associated with large estimated betas. While this procedure is appropriate in the absence of measurement error, this procedure is less appropriate when estimated betas reflect both the true betas and measurement error. Hence, LM suggest constructing portfolios with minimum idiosyncratic risk, with betas of zero w.r.t. all factors other than the factor being tracked, and where the unit-beta constraint is replaced by the constraint that the sum of portfolio weights equals one. LM approximate the estimate 
of the $\hat{\Sigma}_{e e}$ matrix with a diagonal matrix consisting of estimates of the idiosyncratic variances, $D_{\hat{\sigma}_{e}}$. We also consider a further approximation, where all the variances are the same, $I \hat{\sigma}_{e}^{2}$. Note that since the LM portfolios do not have a beta of one, we divide the portfolio excess cash flows by the portfolio beta, to obtain estimates of the factor risk premium. We perform the analysis for both the C-CAPM and the I-CAPM.

Results for the LM portfolios are not encouraging. In the case of the I-CAPM, for example, the LM procedure leads to percentage biases of $6.74 \%(W=I)$ and $24.50 \%(W=$ $\left.\left(D_{\hat{\sigma}_{e}}\right)^{-1}\right)$. For a comparison, the CSR approach leads to percentage biases of $-3.69 \%$ (OLS) and $23.50 \%$ (GLS), while the $\lambda^{\star}$ estimate has a bias of $9.49 \%$. Percentage RMSEs are $72.76 \%$ and $639.97 \%$ for the LM portfolios, $67.44 \%$ and $52.99 \%$ for the unit-beta portfolios, and $55.14 \%$ for the maximum-correlation portfolio. Hence, the LM procedures do not have an obvious advantage over the CSR and maximum-correlation procedures.

\section{Bias corrections}

Chen and Kan (2006) derive the small-sample distribution of the estimates of $\lambda$, for both the OLS and the GLS cases, under the assumption of Gaussian returns. In addition, they show that the adjustments suggested by Litzenberger and Ramaswamy (1979) and Kim (1995), based on asymptotic results, lead to bias-adjusted estimators without finite moments.

We study the small-sample properties of the bias-adjusted estimators proposed by Chen and Kan (2006). We replicate the simulation analysis presented in Table II of the paper, for the case of the i.i.d. bootstrap. Interestingly, the corrections proposed by Chen and Kan are effective at reducing the bias only in eight out of 16 cases. This result differs from Chen and Kan, who find, for the most part, that the bias-adjusted estimates have less bias than the standard estimates in simulations. We attribute the difference in results to the fact that they assume that returns and factors are either multivariate normal or multivariate $t$ distributed, whereas we sample from the empirical distribution. Moreover, the RMSEs of the bias-adjusted estimates increase substantially, especially for the shorter sample of 240 months. For example, in the case of the C-CAPM, size portfolios, $W=I$, the RMSE is 95.33\% for the standard estimate, and $211.91 \%$ for the bias-adjusted estimate. This result is in line with the substantial increase in standard deviation of the bias-adjusted estimates 
documented by Chen and Kan, especially for the CSR-GLS case.

In the comparison between the $\lambda$ and the $\lambda^{\star}$ estimates, the introduction of the biasadjustment means that now there are cases in which the CSR-GLS estimator has less bias than the $\lambda^{\star}$ estimator (without bias-adjustment, the CSR-GLS estimator always has more bias than the $\lambda^{\star}$ estimator). On the other hand, there are cases in which the $\lambda^{\star}$ estimator has less bias than the bias-adjusted CSR-OLS estimator. Finally, the $\lambda^{\star}$ estimates always have a lower RMSE that both the bias-adjusted CSR-OLS and CSR-GLS estimates.

In summary, in terms of bias, the bias-adjustment of Chen and Kan (2006) does not significantly alter the comparison between the LFM and $\mathrm{LFM}^{\star}$ formulations. In terms of RMSE, the bias-adjustment makes the $\lambda$ estimates always noisier than the $\lambda^{\star}$ estimates.

\section{Long-horizon returns}

Some authors have suggested that studying long-horizon returns may help to better uncover the explanatory power of asset-pricing models, especially in the case of the C-CAPM. (see, Lynch, 1996, Daniel and Marshall, 1997, Gabaix and Laibson, 2001, and Jagannathan and Wang, 2005.) Hence, we perform a simulation exercise where we simulate an i.i.d. economy at the monthly frequency, but we study the inference on the factor risk premia using quarterly and annual returns. We perform the simulation for both the C-CAPM and the I-CAPM.

As one would expect, results deteriorate relative to the case of monthly returns. Yet, at least in the case of the C-CAPM, the deterioration is much less marked for the $\lambda^{\star}$ estimates than for the CSR estimates. For example, consider the case of annual returns. The percentage biases in the CSR-OLS and CSR-GLS estimates are $-10.43 \%$ and $-66.55 \%$, respectively. For a comparison, the corresponding percentage biases in the case of monthly returns are $6.40 \%$ and $-18.16 \%$. Similarly, percentage RMSEs are $114.62 \%$ and $70.40 \%$, in the case of annual returns, while they are $52.72 \%$ and $33.29 \%$ in the case of monthly returns. There is much less deterioration in the properties of the $\lambda^{\star}$ estimates. Percentage bias and RMSE are $-0.57 \%$ and $43.28 \%$, respectively, in the case of annual returns. For a comparison, they are $-0.69 \%$ and $36.70 \%$ in the case of monthly returns. Our results indicate that while the use of long-horizon returns may get around issues of mis-alignment between consumption decisions and returns, it may also worsen considerably the properties of the risk-premium estimators, 
particularly those based on the construction of unit-beta portfolios.

\section{E. Portfolio implications}

The previous sections have focused on the implications of the LFM and LFM ${ }^{\star}$ representations for mean estimates of risk premia. It is now interesting to see if the two representations also have different implications for the covariance structure in asset returns for the purpose of constructing mean-variance efficient portfolios. Specifically, we can take an "APT view" of the factors $y_{t}$ and $y_{t}^{\star}$, and approximate the covariance matrices $\Sigma_{e e}$ and $\Sigma_{e^{\star} e^{\star}}$ with $D_{\hat{\sigma}_{e}}$ and $D_{\hat{\sigma}_{e^{\star}}}$. The resulting approximate covariance matrices can then be used as inputs for the construction of mean-variance efficient portfolios.

We construct global minimum-variance portfolios (GMV) and we evaluate their out-ofsample performance. Following the methodology of Jagannathan and Ma (2003), we estimate the covariance matrix of returns at the end of April of each year, using monthly returns of the previous five years. The GMV portfolios are then held for one year. This procedure is repeated from 1964 to 2001. We consider two portfolios based on the restrictions of the LFM and the $\mathrm{LFM}^{\star}$ representations, a portfolio based on the sample covariance matrix, and an equally-weighted portfolio. In addition to the methodology of Jagannathan and Ma, we also consider the case where the GMV portfolios are held only for one month, and where the exercise is repeated every month. Covariance matrices are estimated using either a lengthening sample (initial sample length $=60$ months) with a fixed starting date, or moving windows of 60 and 360 months. In all experiments we consider two sets of base assets: the ten size portfolios and the $25 \mathrm{FF}$ portfolios.

Interestingly, in nine of the ten scenarios, the restrictions of the $\mathrm{LFM}^{\star}$ formulation lead to portfolios with lower volatility than the LFM formulation. Moreover, in six scenarios, the average return of the LFM^ ${ }^{\star}$ portfolios is higher than the average return of the LFM portfolios, while in the remaining four scenarios average returns are essentially the same. Following Jagannathan and Ma (2003), we compute t-ratios on the differences between average returns and average squared returns. In eight of the nine cases where the LFM are less volatile than the LFM portfolios, the difference in average squared returns is significant (absolute t-ratio above two). 
We also compare the performance of the $\mathrm{LFM}^{\star}$ portfolios to portfolios constructed with the unrestricted sample covariance matrix, and to equally-weighted portfolios. In the comparisons with the "unrestricted" portfolios, the LFM^ portfolios always lead to higher standard deviation, and in eight out of ten cases the average return is also lower. Not surprisingly, the better performance of the unrestricted portfolios comes at the "cost" of more extreme positions: in eight cases, the average short interest in the unrestricted portfolios is higher than the average short interest in the $\mathrm{LFM}^{\star}$ portfolios. In comparisons with the equallyweighted portfolios, the $\mathrm{LFM}^{\star}$ portfolios have a lower standard deviation in six cases, while in four cases the average return is higher.

In summary, the LFM^ ${ }^{\star}$ portfolios outperform the LFM portfolios. On the other hand, the $\mathrm{LFM}^{\star}$ portfolios do worse than the unrestricted portfolios, while they perform similarly to the equally-weighted portfolios.

\section{Conclusions}

This paper considers two alternative formulations of the linear factor model with non-traded factors. The first formulation is the traditional one, where we estimate risk premia and alphas by means of a cross-sectional regression of average returns on betas. The second formulation replaces the factors with their projections onto the augmented span of excess returns. This second formulation requires only time-series regressions for the estimation of risk premia and alphas. We compare the theoretical properties of the two approaches and perform a simulation exercise to assess the small-sample properties of the estimates.

We find that the $\mathrm{LFM}^{\star}$ formulation of the model leads to estimates of risk premia with less bias than, although similar RMSEs to, the CSR-GLS estimates. Since this pattern is robust to the different simulation scenarios considered, we believe that it is not an artifact of our experimental design. Moreover, although we do not cast our analysis within an explicit decision-theoretic framework, it is natural to think that the choice of the estimator depends on the loss function of the agent using the estimates. If the agent is only concerned with the first moment of the estimates, then the choice falls on the $\lambda^{\star}$ estimator. On the other hand, if the agent cares about first and second moments, then the choice depends on the 
trade-off between bias and variability. The RMSE $\left(=\sqrt{\text { variance }+ \text { bias }^{2}}\right)$ places equal weight on variance and squared bias. Hence, an agent equally concerned about variability and bias would be roughly indifferent between the two methods.

We also show that the difference in bias between the two estimators is gradually reduced as the non-traded factor explains more of the variability of returns. On the other hand, in the case of a single factor observed with noise, the LFM^ ${ }^{\star}$ risk-premium estimate works much better than the CSR estimates, both in terms of bias and RMSE.

Under the null, rejection rates are roughly correct for the LFM formulation, but too high for the $\mathrm{LFM}^{\star}$ formulation. Hence, testing the multi-beta model in its $\mathrm{LFM}^{\star}$ formulation requires an adjustment of critical values, based on the empirical distribution of the statistics under the null. With the bootstrap correction, the power of tests of multi-beta models is similar in the LFM^ and CSR-GLS formulations, and higher than in the CSR-OLS approach.

In light of these results, we conclude that when estimating risk premia and testing multibeta models, the $\mathrm{LFM}^{\star}$ formulation should be considered in addition to, or even instead of, the more traditional LFM formulation. 


\section{Appendix}

Proof of Result 1. We have

$$
\begin{aligned}
E\left(r_{t}\right) & =\alpha^{\star}+\left(\beta^{\star}\right)^{\top} E\left(y^{\star}\right) \\
& =\alpha^{\star}+\left(\beta^{\star}\right)^{\top}\left(\gamma^{\star}\right)^{\top} E\left(r_{t}\right) \\
& =\alpha^{\star}+\left(\beta^{\star}\right)^{\top}\left(\gamma^{\star}\right)^{\top} \alpha+\left(\beta^{\star}\right)^{\top}\left(\gamma^{\star}\right)^{\top} \beta^{\top} \lambda .
\end{aligned}
$$

We have

$$
\begin{aligned}
\left(\beta^{\star}\right)^{\top}\left(\gamma^{\star}\right)^{\top} \beta^{\top} & =\Sigma_{r r} \gamma^{\star}\left(\left(\gamma^{\star}\right)^{\top} \Sigma_{r r} \gamma^{\star}\right)^{-1}\left(\gamma^{\star}\right)^{\top} \Sigma_{r y} \Sigma_{y y}^{-1} \\
& =\Sigma_{r r} \Sigma_{r r}^{-1} \Sigma_{r y}\left(\Sigma_{y r} \Sigma_{r r}^{-1} \Sigma_{r r} \Sigma_{r r}^{-1} \Sigma_{r y}\right)^{-1} \Sigma_{y r} \Sigma_{r r}^{-1} \Sigma_{r y} \Sigma_{y y}^{-1} \\
& =\Sigma_{r y}\left(\Sigma_{y r} \Sigma_{r r}^{-1} \Sigma_{r y}\right)^{-1} \Sigma_{y r} \Sigma_{r r}^{-1} \Sigma_{r y} \Sigma_{y y}^{-1} \\
& =\Sigma_{r y} \Sigma_{y y}^{-1} \\
& =\beta^{\top} .
\end{aligned}
$$

Hence, we write

$$
E\left(r_{t}\right)-\left(\beta^{\star}\right)^{\top}\left(\gamma^{\star}\right)^{\top} \beta^{\top} \lambda=E\left(r_{t}\right)-\beta^{\top} \lambda=\alpha=\alpha^{\star}+\left(\beta^{\star}\right)^{\top}\left(\gamma^{\star}\right)^{\top} \alpha
$$

and

$$
\alpha-\left(\beta^{\star}\right)^{\top}\left(\gamma^{\star}\right)^{\top} \alpha=\alpha^{\star}
$$

It is straightforward to show that both $I-\left(\beta^{\star}\right)^{\top}\left(\gamma^{\star}\right)^{\top}$ and $\left(\beta^{\star}\right)^{\top}\left(\gamma^{\star}\right)^{\top}$ are idempotent matrices. What we want to show is that if $\left(\beta^{\star}\right)^{\top}\left(\gamma^{\star}\right)^{\top}$ is idempotent of rank $K$, then $I-\left(\beta^{\star}\right)^{\top}\left(\gamma^{\star}\right)^{\top}$ is idempotent of rank $N-K$. Notice that $\left(\beta^{\star}\right)^{\top}\left(\gamma^{\star}\right)^{\top}$ can be written as $\Sigma_{r y}\left(\Sigma_{y r} \Sigma_{r r}^{-1} \Sigma_{r y}\right)^{-1} \Sigma_{y r} \Sigma_{r r}^{-1}$. Given that $\Sigma_{r r}^{-1}$ is of full rank

$$
\operatorname{rank}\left[\Sigma_{r y}\left(\Sigma_{y r} \Sigma_{r r}^{-1} \Sigma_{r y}\right)^{-1} \Sigma_{y r} \Sigma_{r r}^{-1}\right]=\operatorname{rank}\left[\Sigma_{r y}\left(\Sigma_{y r} \Sigma_{r r}^{-1} \Sigma_{r y}\right)^{-1} \Sigma_{y r}\right]
$$

Since $\Sigma_{y r} \Sigma_{r r}^{-1} \Sigma_{r y}$ is symmetric and invertible, $\operatorname{rank}\left[\left(\beta^{\star}\right)^{\top}\left(\gamma^{\star}\right)^{\top}\right]=\operatorname{rank}\left[\Sigma_{r y}\left(\Sigma_{y r} \Sigma_{r r}^{-1} \Sigma_{r y}\right)^{-1} \Sigma_{y r}\right]=$ $\operatorname{rank}\left(\Sigma_{y r}\right)=K$. Now, the null space of $I-\left(\beta^{\star}\right)^{\top}\left(\gamma^{\star}\right)^{\top}$ is the set of all $z \neq 0$ such that $\left[I-\left(\beta^{\star}\right)^{\top}\left(\gamma^{\star}\right)^{\top}\right] z=0$. But $\left[I-\left(\beta^{\star}\right)^{\top}\left(\gamma^{\star}\right)^{\top}\right] z=0$ if and only if $z=\left(\beta^{\star}\right)^{\top}\left(\gamma^{\star}\right)^{\top} z$, i.e., 
$z$ is an eigenvector of $\left(\beta^{\star}\right)^{\top}\left(\gamma^{\star}\right)^{\top}$ with eigenvalue equal to 1 . Since $\left(\beta^{\star}\right)^{\top}\left(\gamma^{\star}\right)^{\top}$ is idempotent, the dimension of all such eigenvectors is equal to $\operatorname{rank}\left[\left(\beta^{\star}\right)^{\top}\left(\gamma^{\star}\right)^{\top}\right]=K$, which means that $\operatorname{rank}\left[I-\left(\beta^{\star}\right)^{\top}\left(\gamma^{\star}\right)^{\top}\right]=N-K$. Moreover, the null space of the idempotent matrix $I-\left(\beta^{\star}\right)^{\top}\left(\gamma^{\star}\right)^{\top}$ is spanned by linear combinations of the column vectors in $\beta^{\top}$. Since $\beta^{\top}$ is of $\operatorname{rank} K$, the set of all $\alpha=\beta^{\top} \delta$ for $\delta \in \Re^{K}$ is of dimension $K$. This set is contained in the null space of $I-\left(\beta^{\star}\right)^{\top}\left(\gamma^{\star}\right)^{\top}$ and it must be all of the null space. (We thank Raymond Kan for pointing out this issue.)

A proof of Result 1 for the case where the projection does not contain a constant is in a separate appendix available from the authors upon request.

Proof of Result 2. We have

$$
\lambda^{\star}=\left(\gamma^{\star}\right)^{\top} E\left(r_{t}\right)=\left(\gamma^{\star}\right)^{\top} \alpha+\left(\gamma^{\star}\right)^{\top} \beta^{\top} \lambda=\left(\gamma^{\star}\right)^{\top} \alpha+\Sigma_{y r} \Sigma_{r r}^{-1} \Sigma_{r y} \Sigma_{y y}^{-1} \lambda
$$

Note that

$$
\operatorname{Var}\left(\left(\gamma^{\star}\right)^{\top} r_{t}\right)=\Sigma_{y^{\star} y^{\star}}=\Sigma_{y r} \Sigma_{r r}^{-1} \Sigma_{r r} \Sigma_{r r}^{-1} \Sigma_{r y}=\Sigma_{y r} \Sigma_{r r}^{-1} \Sigma_{r y}
$$

Hence

$$
\lambda^{\star}=\left(\gamma^{\star}\right)^{\top} \alpha+\left(\gamma^{\star}\right)^{\top} \beta^{\top} \lambda=\left(\gamma^{\star}\right)^{\top} \alpha+\Sigma_{y^{\star} y^{\star}} \Sigma_{y y}^{-1} \lambda .
$$

Proof of Result 3. Using the fact that $\tilde{\gamma}$ is the same for $W=\Sigma_{e e}^{-1}$ and $W=\Sigma_{r r}^{-1}$, we have

$$
\begin{aligned}
\beta^{\top}\left(\beta \Sigma_{e e}^{-1} \beta^{\top}\right)^{-1} \beta \Sigma_{e e}^{-1} E\left(r_{t}\right) & =\Sigma_{r y} \Sigma_{y y}^{-1}\left(\beta \Sigma_{r r}^{-1} \beta^{\top}\right)^{-1} \beta \Sigma_{r r}^{-1} E\left(r_{t}\right) \\
& =\Sigma_{r y} \Sigma_{y y}^{-1}\left(\Sigma_{y y}^{-1} \Sigma_{y r} \Sigma_{r r}^{-1} \Sigma_{r y} \Sigma_{y y}^{-1}\right)^{-1} \Sigma_{y y}^{-1} \Sigma_{y r} \Sigma_{r r}^{-1} E\left(r_{t}\right) \\
& =\Sigma_{r y}\left(\Sigma_{y r} \Sigma_{r r}^{-1} \Sigma_{r y}\right)^{-1} \Sigma_{y r} \Sigma_{r r}^{-1} E\left(r_{t}\right) \\
& =\left(\beta^{\star}\right)^{\top} \lambda^{\star} .
\end{aligned}
$$

This implies that when $W=\Sigma_{e e}^{-1}$, then $\beta^{\top} \tilde{\lambda}=\left(\beta^{\star}\right)^{\top} \lambda^{\star}$. Hence, we also have $\tilde{\alpha}=E\left(r_{t}\right)-$ $\beta^{\top} \tilde{\lambda}=E\left(r_{t}\right)-\left(\beta^{\star}\right)^{\top} \lambda^{\star}=\alpha^{\star}$. 


\section{References}

[1] Affleck-Graves, J. F., and D. J. Bradfield, 1993, An examination of the power of univariate tests of the CAPM: A simulation approach, Journal of Economics and Business $45,17-33$.

[2] Amsler, C. E., and P. Schmidt, 1985, A Monte Carlo investigation of the accuracy of multivariate CAPM tests, Journal of Financial Economics 14, 359-375.

[3] Asgharian, H., 2004, A comparative analysis of ability of mimicking portfolios in representing the background factors, working paper, Lund University.

[4] Asgharian, H., and B. Hansson, 2005, A critical investigation of the explanatory role of factor mimicking portfolios in equilibrium pricing, Apllied Financial Economics 15, $835-847$.

[5] Black, F., M. C. Jensen, and M. Scholes, 1972, The capital asset pricing model: Some empirical findings, in M. C. Jensen (ed.), Studies in the Theory of Capital Markets, Praeger, New York.

[6] Brav, A., G. M. Constantinides, and C. C. Geczy, 2002, Asset pricing with heterogeneous consumers and limited participation: empirical evidence, Journal of Political Economy 110, 793-824.

[7] Breeden, D. T., 1979, An intertemporal asset pricing model with stochastic consumption and investment opportunities, Journal of Financial Economics 7, 265-296.

[8] Breeden, D. T., M. R. Gibbons, and R. H. Litzenberger, 1989, Empirical tests of the consumption-oriented CAPM, Journal of Finance 44, 231-262.

[9] Chan, L. K. C., J. Karceski, and J. Lakonishok, 1998, The risk and return from factors, Journal of Financial and Quantitative Analysis 33, 159-188.

[10] Chen R., and R. Kan, 2006, Finite sample analysis of two-pass cross-sectional regressions, working paper, University of Toronto. 
[11] Cochrane, H. J., 2001, Asset pricing, Princeton University Press, Princeton NJ.

[12] Daniel, K., and D. Marshall, 1997, Equity premium and risk-free-rate puzzles at long horizons, Macroeconomic Dynamics 1, 452-484.

[13] Dickey, J. M., 1967, Matricvariate generalizations of the multivariate $t$ distribution and the inverted multivariate $t$ distribution, The Annals of Mathematical Statistics 38, 511518.

[14] Fama, E.F., 1996, Multifactor portfolio efficiency and multifactor asset pricing, Journal of Financial and Quantitative Analysis 31, 441-465.

[15] Fama, E.F., and K. R. French, 1993, Common risk factors in the returns on bonds and stocks, Journal of Financial Economics 33, 3-56.

[16] Fama, E. F., and J. D. MacBeth, 1973, Risk, return and equilibrium, Journal of Political Economy 81, 607-636.

[17] Ferson, W. E., and C. R. Harvey, 1991, The variation of economic risk premiums, Journal of Political Economy 99, 385-415.

[18] Ferson, W. E., and C. R. Harvey, 1999, Conditioning variables and the cross-section of stock returns, Journal of Finance 54, 1325-1360.

[19] Ferson, W. E., A. F. Siegel, and P. T. Xu, 2005, Mimicking portfolios with conditioning information, forthcoming in the Journal of Financial and Quantitative Analysis.

[20] Gabaix, X., and D. Laibson, 2001, The 6D bias and the equity premium puzzle, NBER Macroeconomics Annual, Ben Bernanke and Kenneth Rogoff eds., 257-311.

[21] Hansen, L. P., 1982, Large sample properties of generalized method of moments estimators, Econometrica 50, 1029-54.

[22] Huberman, G., S. Kandel, and R. F. Stambaugh, 1987, Mimicking portfolios and exact arbitrage pricing, Journal of Finance 42, 1-9. 
[23] Jacobs, K., and K. Q. Wang, 2004, Idiosyncratic consumption risk and the cross-section of asset returns, Journal of Finance 59, 2211-2252.

[24] Jagannathan, R., and Z. Wang, 2002, Empirical evaluation of asset pricing models: A comparison of the SDF and Beta methods, Journal of Finance 57, 2337-2367.

[25] Jagannathan, R., and Y. Wang, 2005, Consumption risk and the cost of equity capital, working paper, National Bureau of Economic Research.

[26] Jagannathan, R., and T. Ma, 2003, Risk reduction in large portfolios: Why imposing the wrong constraints helps, Journal of Finance 58, 1651-1683.

[27] Kan, R., and C. Zhang, 1999, Two-pass tests of asset pricing models with useless factors, Journal of Finance 54, 204-235.

[28] Kan, R., and G. Zhou, 1999, A critique of the stochastic discount factor methodology, Journal of Finance 54, 1221-1248.

[29] Kan, R., and G. Zhou, 2001a, Empirical asset pricing: the beta method versus the stochastic discount factor method, working paper, University of Toronto.

[30] Kan, R., and G. Zhou, 2001b, Tests of mean-variance spanning, working paper, University of Toronto.

[31] Kim, D., 1995, The errors in the variables problem in the cross-section of expected stock returns, Journal of Finance 50, 1605-1634.

[32] Kimmel, R. L., 2003, Risk premia in linear factor models: theoretical and econometric issues, working paper, Princeton University.

[33] Lamont, O., 2001, Economic tracking portfolios, Journal of Econometrics 105, 161-184.

[34] Lehmann, B., and D.M. Modest, 1988, The empirical foundations of the arbitrage pricing theory, Journal of Financial Economics 21, 213-254.

[35] Lynch, A., 1996, Decision frequency and synchronization across agents: Implications for aggregate consumption and equity return, Journal of Finance 51, 1479-1498. 
[36] Litzenberger, R. H., and K. Ramaswamy, 1979, The effect of personal taxes and dividends on capital asset prices, Journal of Financial Economics 7, 163-195.

[37] MacKinlay, A. C., 1987, On multivariate tests of the CAPM, Journal of Financial Economics 18, 341-372.

[38] Merton, R., 1973, An intertemporal capital asset pricing model, Econometrica 41, 867887.

[39] Newey, W., and K. West, 1987, A simple positive definite heteroskedasticity and autocorrelation consistent covariance matrix, Econometrica 55, 703-708.

[40] Peñaranda, F., and E. Sentana, 2004, Spanning tests in return and stochastic discount mean-variance frontiers: A unifying approach, working paper, CEMFI.

[41] Shanken, J., 1985, Multivariate tests of the zero-beta CAPM, Journal of Financial Economics 14, 327-348.

[42] Shanken, J., 1992, On the estimation of beta-pricing models, Review of Financial Studies $5,1-33$.

[43] Shanken, J., and G. Zhou, 2006, Estimating and testing beta pricing models: Alternative methods and their performance in simulations, forthcoming in the Journal of Financial Economics. 


\section{Table I: LFM vs. LFM $^{\star}$ representation}

We estimate a C-CAPM and an I-CAPM model. Parameter estimates of the LFM are obtained by cross-sectional regression, with $W=I$ (OLS) and $W=\Sigma_{e e}^{-1}$ (GLS). Estimates of the parameters of the $\mathrm{LFM}^{\star}$ are obtained by time-series regressions. Asymptotic standard errors and $\chi^{2}$ statistics are obtained by GMM, assuming no serial dependence. The sample is from 03:1959 to 2002:11. We consider the ten size portfolios as the test assets.

\section{Panel A: LFM}

\begin{tabular}{|c|c|c|c|c|c|c|c|c|}
\hline & \multicolumn{4}{|c|}{ C-CAPM } & \multicolumn{4}{|c|}{ I-CAPM } \\
\hline & $\lambda_{O L S}$ & $\lambda_{G L S}$ & $\chi_{O L S}^{2}$ & $\chi_{G L S}^{2}$ & $\lambda_{O L S}$ & $\lambda_{G L S}$ & $\chi_{O L S}^{2}$ & $\chi_{G L S}^{2}$ \\
\hline Estimate & 0.24 & 0.09 & 17.94 & 23.92 & -0.47 & -0.33 & 18.14 & 18.81 \\
\hline$t$-ratio & 2.23 & 1.43 & & & -1.63 & -1.18 & & \\
\hline$p$-value & 0.026 & 0.152 & 0.036 & 0.004 & 0.104 & 0.238 & 0.034 & 0.027 \\
\hline
\end{tabular}

Panel B: LFM*

\begin{tabular}{lccccc} 
& \multicolumn{2}{c}{ C-CAPM } & & \multicolumn{2}{c}{ I-CAPM } \\
\cline { 2 - 3 } \cline { 5 - 5 } & $\lambda^{*}$ & $\chi^{2}$ & & $\lambda^{*}$ & $\chi^{2}$ \\
\hline Estimate & 0.01 & 28.66 & & -0.01 & 23.19 \\
$t$-ratio & 1.03 & & -1.19 & \\
$p$-value & 0.30 & 0.001 & & 0.23 & 0.006 \\
\hline
\end{tabular}




\section{Table II: C-CAPM; $\lambda$ vs $\lambda^{\star}$}

We simulate a one-factor C-CAPM economy 10,000 times under the null (the $\alpha$ parameters are set to zero). Parameter estimates of the LFM are obtained using GMM with $W=I$. In the bootstrap (i.i.d. and block), we simulate jointly the factor and the residuals from the estimated LFM. We consider two lengths of the data set: the full sample of 525 observations (full sample) and a shorter sample of 240 observations (240 months). We consider two choices of assets, the ten size portfolios (size) and the 25 Fama-French portfolios (FF). When we investigate the properties of estimates of the LFM, we consider estimates based on the two weighting matrices $W=I$ and $W=s e e=\Sigma_{e e}^{-1}$. Estimates of the parameters of the $\mathrm{LFM}^{\star}$ are obtained by exactly-identified GMM. Asymptotic statistics are obtained assuming no serial correlation in the i.i.d bootstrap and serial correlation of order 3 (Newey-West adjustment) in the block bootstrap. For each panel, the first row reports the population value of the risk premium as a percentage of the population standard deviation of the excess return on the corresponding mimicking portfolio; the second row reports the average value across simulations of the risk premium as a percentage of the population standard deviation of the excess return on the corresponding mimicking portfolio; the third and fourth rows report absolute (percentage) bias and absolute (percentage) root mean square errors (RMSE), respectively; the fifth and sixth rows, in the order, report mean and standard deviation of the t-ratios across simulations; finally, the seventh row reports the ratio between average asymptotic standard errors across simulations and empirical standard deviation. 
Panel A: Finite Sample properties of $\lambda$ (IID Bootstrap)

\begin{tabular}{|c|c|c|c|c|c|c|c|c|}
\hline & \multicolumn{4}{|c|}{ Full Sample } & \multicolumn{4}{|c|}{240 Months } \\
\hline & \multicolumn{2}{|c|}{ size } & \multicolumn{2}{|c|}{ FF } & \multicolumn{2}{|c|}{ size } & \multicolumn{2}{|c|}{ FF } \\
\hline & $\mathbf{W}=\mathbf{I}$ & $\mathbf{W}=\mathrm{sse}$ & $\mathbf{W}=\mathbf{I}$ & $\mathbf{W}=\mathrm{sse}$ & $\mathbf{W}=\mathbf{I}$ & $\mathbf{W}=$ sse & $\mathbf{W}=\mathbf{I}$ & $\mathbf{W}=\mathrm{sse}$ \\
\hline$\lambda$ & 11.57 & 15.86 & 11.73 & 17.96 & 11.57 & 15.86 & 11.73 & 17.96 \\
\hline$\hat{\lambda}$ & 12.31 & 12.98 & 12.12 & 11.17 & 13.08 & 10.44 & 12.43 & 7.35 \\
\hline Bias & $\begin{array}{c}0.74 \\
(6.40)\end{array}$ & $\begin{array}{c}-2.88 \\
(-18.16)\end{array}$ & $\begin{array}{c}0.39 \\
(3.32)\end{array}$ & $\begin{array}{c}-6.79 \\
(-37.81)\end{array}$ & $\begin{array}{c}1.51 \\
(13.05)\end{array}$ & $\begin{array}{c}-5.42 \\
(-34.17)\end{array}$ & $\begin{array}{c}0.70 \\
(5.97)\end{array}$ & $\begin{array}{c}-10.62 \\
(-59.08)\end{array}$ \\
\hline$R M S E$ & $\begin{array}{c}6.10 \\
(52.72)\end{array}$ & $\begin{array}{c}5.28 \\
(33.29)\end{array}$ & $\begin{array}{c}5.48 \\
(46.72)\end{array}$ & $\begin{array}{c}7.90 \\
(43.99)\end{array}$ & $\begin{array}{c}11.03 \\
(95.33)\end{array}$ & $\begin{array}{c}8.11 \\
(51.13)\end{array}$ & $\begin{array}{c}8.63 \\
(73.57)\end{array}$ & $\begin{array}{c}11.72 \\
(65.26)\end{array}$ \\
\hline$t_{\text {mean }}$ & -0.12 & -0.78 & -0.12 & -1.83 & -0.18 & -1.08 & -0.18 & -2.39 \\
\hline$t_{\text {std }}$ & 0.97 & 1.07 & 0.97 & 1.17 & 0.93 & 1.10 & 0.93 & 1.24 \\
\hline$\frac{\text { AsySE }}{E m p S t d}$ & 0.97 & 0.98 & 0.98 & 0.99 & 0.96 & 0.95 & 1.01 & 0.94 \\
\hline
\end{tabular}

Panel B: Finite Sample properties of $\lambda$ (Block Bootstrap)

\begin{tabular}{|l||c|c|c|c|c|cc|c|c|}
\hline \multicolumn{1}{|c||}{} & \multicolumn{4}{c|}{ Full Sample } & \multicolumn{4}{c|}{ 240 Months } \\
\hline \multicolumn{1}{|c||}{} & \multicolumn{2}{c|}{ size } & \multicolumn{2}{c|}{ FF } & \multicolumn{2}{c|}{ size } & \multicolumn{2}{c|}{ FF } \\
\hline \multirow{2}{*}{ W=I } & W=sse & W=I & W=sse & W=I & W=sse & W=I & W=sse \\
\hline$\lambda$ & 11.57 & 15.86 & 11.73 & 17.96 & 11.57 & 15.86 & 11.73 & 17.96 \\
$\hat{\lambda}$ & 12.10 & 12.87 & 12.00 & 11.22 & 13.06 & 10.47 & 12.54 & 7.38 \\
Bias & 0.53 & -2.99 & 0.27 & -6.74 & 1.49 & -5.39 & 0.81 & -10.58 \\
RMSE & $(4.58)$ & $(-18.85)$ & $(2.30)$ & $(-37.53)$ & $(12.88)$ & $(-33.98)$ & $(6.91)$ & $(-58.91)$ \\
& 6.20 & 5.43 & 5.66 & 7.90 & 11.07 & 8.16 & 9.22 & 11.73 \\
$t_{\text {mean }}$ & $(53.59)$ & $(34.24)$ & $(48.25)$ & $(43.99)$ & $(95.68)$ & $(51.45)$ & $(78.60)$ & $(65.31)$ \\
$t_{\text {std }}$ & -0.12 & -0.78 & -0.13 & -1.79 & -0.15 & -1.05 & -0.15 & -2.38 \\
AsySE & 0.98 & 1.07 & 0.98 & 1.16 & 0.94 & 1.10 & 0.94 & 1.25 \\
EmpStd & 0.97 & 0.99 & 0.99 & 0.98 & 0.96 & 0.96 & 0.99 & 0.93 \\
\hline
\end{tabular}

sse $=\hat{\Sigma}_{e e}^{-1}$ 
Panel C: Finite Sample properties of $\lambda^{\star}$

\begin{tabular}{|l||ccc|c|c|c|c|c|c|}
\hline \multicolumn{1}{|c||}{} & \multicolumn{4}{c|}{ IID Bootstrap } & \multicolumn{4}{c|}{ Block Bootstrap } \\
\hline \multicolumn{1}{|||}{} & Full Sample & \multicolumn{2}{c|}{ 240 Months } & Full Sample & \multicolumn{2}{c|}{ 240 Months } \\
\hline \multicolumn{1}{|c||}{ size } & FF & size & FF & Size & FF & Size & FF \\
\hline$\lambda^{\star}$ & 15.86 & 17.96 & 15.86 & 17.96 & 15.86 & 17.96 & 15.86 & 17.96 \\
$\hat{\lambda}^{\star}$ & 15.75 & 16.99 & 15.59 & 16.01 & 15.58 & 17.04 & 15.55 & 15.99 \\
Bias & -0.11 & -0.97 & -0.27 & -1.95 & -0.28 & -0.92 & -0.31 & -1.97 \\
RMSE & $-0.69)$ & $(-5.40)$ & $(-1.70)$ & $(-10.86)$ & $(-1.77)$ & $(-5.12)$ & $(-1.95)$ & $(-10.97)$ \\
$t_{\text {mean }}$ & $(36.70)$ & $(35.52)$ & $(58.76)$ & $(62.97)$ & $(37.39)$ & $(37.42)$ & $(6.52$ & 11.45 \\
$t_{\text {std }}$ & -0.12 & -0.13 & -0.12 & -0.20 & -0.14 & -0.21 & -0.13 & -0.21 \\
AsySE & 0.97 & 0.92 & 0.92 & 0.89 & 0.98 & 0.96 & 0.93 & 0.91 \\
EmpStd & 1.05 & 1.09 & 1.09 & 1.11 & 1.04 & 1.05 & 1.07 & 1.10 \\
\hline
\end{tabular}


Table III: I-CAPM; $\lambda$ vs $\lambda^{\star}$

We simulate a two-factor I-CAPM economy 10,000 times under the null (the $\alpha$ parameters are set to zero). The two factors are the VW NYSE/AMEX/NASDAQ index return and the change in the dividend yield. Parameter estimates of the LFM are obtained using GMM with $W=I$. In the bootstrap (i.i.d. and block), we simulate jointly the factors and the residuals from the estimated LFM. We consider two lengths of the data set: the full sample of 525 observations (full sample) and a shorter sample of 240 observations (240 months). We consider two choices of assets, the ten size portfolios (size) and the 25 Fama-French portfolios (FF). When we investigate the properties of estimates of the LFM, we consider estimates based on the two weighting matrices $W=I$ and $W=$ see $=\Sigma_{e e}^{-1}$. Estimates of the parameters of the LFM $^{\star}$ are obtained by exactly-identified GMM. Asymptotic statistics are obtained assuming no serial correlation in the i.i.d bootstrap and serial correlation of order 3 (Newey-West adjustment) in the block bootstrap. For each panel, the first row reports the population value of the risk premium on the non-traded factor as a percentage of the population standard deviation of the excess return on the corresponding mimicking portfolio (see Section VII.A); the second row reports the average value across simulations of the risk premium on the non-traded factor as a percentage of the population standard deviation of the excess return on the corresponding mimicking portfolio; the third and fourth rows report absolute (percentage) bias and absolute (percentage) root mean square errors (RMSE), respectively; the fifth and sixth rows, in the order, report mean and standard deviation of the t-ratios across simulations; finally, the seventh row reports the ratio between average asymptotic standard errors across simulations and empirical standard deviation. 
Panel A: Finite Sample properties of $\lambda$ (IID Bootstrap)

\begin{tabular}{|c|c|c|c|c|c|c|c|c|}
\hline & \multicolumn{4}{|c|}{ Full Sample } & \multicolumn{4}{|c|}{240 Months } \\
\hline & \multicolumn{2}{|c|}{ size } & \multicolumn{2}{|c|}{ FF } & \multicolumn{2}{|c|}{ size } & \multicolumn{2}{|c|}{ FF } \\
\hline & $\mathbf{W}=\mathbf{I}$ & $\mathrm{W}=\mathrm{sse}$ & $\mathbf{W}=\mathbf{I}$ & $\mathrm{W}=\mathrm{sse}$ & $\mathbf{W}=\mathbf{I}$ & $\mathrm{W}=\mathrm{sse}$ & $\mathbf{W}=\mathbf{I}$ & $\mathrm{W}=\mathrm{sse}$ \\
\hline$\lambda$ & -7.31 & -8.85 & -9.36 & -16.92 & -7.31 & -8.85 & -9.36 & -16.92 \\
\hline$\hat{\lambda}$ & -7.58 & -6.77 & -9.28 & -11.94 & -8.03 & -4.93 & -8.77 & -8.28 \\
\hline Bias & $\begin{array}{c}-0.27 \\
(-3.69)\end{array}$ & $\begin{array}{c}2.08 \\
(23.50)\end{array}$ & $\begin{array}{c}0.08 \\
(0.85)\end{array}$ & $\begin{array}{c}4.98 \\
(29.43)\end{array}$ & $\begin{array}{c}-0.72 \\
(-9.85)\end{array}$ & $\begin{array}{c}3.92 \\
(44.29)\end{array}$ & $\begin{array}{c}0.59 \\
(6.30)\end{array}$ & $\begin{array}{c}8.64 \\
(51.06)\end{array}$ \\
\hline$R M S E$ & $\begin{array}{c}4.93 \\
(67.44)\end{array}$ & $\begin{array}{c}4.69 \\
(52.99)\end{array}$ & $\begin{array}{c}4.93 \\
(52.67)\end{array}$ & $\begin{array}{c}6.52 \\
(38.53)\end{array}$ & $\begin{array}{c}8.25 \\
(113.85)\end{array}$ & $\begin{array}{c}6.85 \\
(77.40)\end{array}$ & $\begin{array}{c}6.94 \\
(74.15)\end{array}$ & $\begin{array}{l}10.30 \\
(60.87)\end{array}$ \\
\hline$t_{\text {mean }}$ & 0.11 & 0.61 & 0.22 & 1.31 & 0.15 & 0.83 & 0.33 & 1.78 \\
\hline$t_{s t d}$ & 0.96 & 1.04 & 0.98 & 1.12 & 0.93 & 1.04 & 0.98 & 1.19 \\
\hline$\frac{\text { AsySE }}{\text { EmpStd }}$ & 0.98 & 0.99 & 1.02 & 0.97 & 0.97 & 0.99 & 1.04 & 0.92 \\
\hline
\end{tabular}

Panel B: Finite Sample properties of $\lambda$ (Block Bootstrap)

\begin{tabular}{|c|c|c|c|c|c|c|c|c|}
\hline & \multicolumn{4}{|c|}{ Full Sample } & \multicolumn{4}{|c|}{240 Months } \\
\hline & \multicolumn{2}{|c|}{ size } & \multicolumn{2}{|c|}{ FF } & \multicolumn{2}{|c|}{ size } & \multicolumn{2}{|c|}{ FF } \\
\hline & $\mathbf{W}=\mathbf{I}$ & $\mathbf{W}=\mathbf{s s e}$ & $\mathbf{W}=\mathbf{I}$ & $\mathrm{W}=\mathrm{sse}$ & $\mathbf{W}=\mathbf{I}$ & $\mathrm{W}=\mathrm{sse}$ & $\mathbf{W}=\mathbf{I}$ & $\mathrm{W}=\mathrm{sse}$ \\
\hline$\lambda$ & -7.31 & -8.85 & -9.36 & -16.92 & -7.31 & -8.85 & -9.36 & -16.92 \\
\hline$\hat{\lambda}$ & -7.47 & -6.78 & -9.20 & -11.96 & -7.70 & -4.97 & -8.68 & -8.20 \\
\hline Bias & $\begin{array}{l}-0.16 \\
(-2.19)\end{array}$ & $\begin{array}{c}2.07 \\
(23.39)\end{array}$ & $\begin{array}{c}0.16 \\
(1.71)\end{array}$ & $\begin{array}{c}4.96 \\
(29.31)\end{array}$ & $\begin{array}{l}-0.39 \\
(-5.33)\end{array}$ & $\begin{array}{c}3.88 \\
(43.84)\end{array}$ & $\begin{array}{l}0.68 \\
(7.26)\end{array}$ & $\begin{array}{c}8.72 \\
(51.54)\end{array}$ \\
\hline$R M S E$ & $\begin{array}{c}5.10 \\
(69.77)\end{array}$ & $\begin{array}{c}4.84 \\
(54.69)\end{array}$ & $\begin{array}{c}5.16 \\
(55.13)\end{array}$ & $\begin{array}{c}6.55 \\
(38.71)\end{array}$ & $\begin{array}{c}8.51 \\
(116.42)\end{array}$ & $\begin{array}{c}6.99 \\
(78.98)\end{array}$ & $\begin{array}{c}7.30 \\
(77.99)\end{array}$ & $\begin{array}{l}10.38 \\
(61.35)\end{array}$ \\
\hline$t_{\text {mean }}$ & 0.10 & 0.60 & 0.22 & 1.30 & 0.15 & 0.82 & 0.33 & 1.82 \\
\hline$t_{s t d}$ & 0.98 & 1.04 & 1.01 & 1.12 & 0.94 & 1.04 & 1.01 & 1.21 \\
\hline$\frac{\text { Asy.se }}{\text { Emp.Std }}$ & 0.97 & 0.98 & 0.99 & 0.95 & 0.94 & 0.97 & 1.00 & 0.90 \\
\hline
\end{tabular}

sse $=\hat{\Sigma}_{e e}^{-1}$ 
Panel C: Finite Sample properties of $\lambda^{\star}$

\begin{tabular}{|c|c|c|c|c|c|c|c|c|}
\hline & \multicolumn{4}{|c|}{ IID Bootstrap } & \multicolumn{4}{|c|}{ Block Bootstrap } \\
\hline & \multicolumn{2}{|c|}{ Full Sample } & \multicolumn{2}{|c|}{240 Months } & \multicolumn{2}{|c|}{ Full Sample } & \multicolumn{2}{|c|}{240 Months } \\
\hline & size & FF & size & FF & Size & FF & Size & FF \\
\hline$\lambda^{\star}$ & -8.85 & -16.92 & -8.85 & -16.92 & -8.85 & -16.92 & -8.85 & -16.92 \\
\hline$\hat{\lambda}^{\star}$ & -8.01 & -15.50 & -7.06 & -13.99 & -8.04 & -15.52 & -7.11 & -13.93 \\
\hline Bias & $\begin{array}{c}0.84 \\
(9.49)\end{array}$ & $\begin{array}{l}1.42 \\
(8.39)\end{array}$ & $\begin{array}{c}1.79 \\
(20.22)\end{array}$ & $\begin{array}{c}2.93 \\
(17.32)\end{array}$ & $\begin{array}{c}0.81 \\
(9.15)\end{array}$ & $\begin{array}{l}1.40 \\
(8.27)\end{array}$ & $\begin{array}{c}1.74 \\
(19.66)\end{array}$ & $\begin{array}{c}2.99 \\
(17.67)\end{array}$ \\
\hline$R M S E$ & $\begin{array}{c}4.88 \\
(55.14)\end{array}$ & $\begin{array}{c}5.66 \\
(33.45)\end{array}$ & $\begin{array}{c}8.19 \\
(92.54)\end{array}$ & $\begin{array}{c}9.92 \\
(58.63)\end{array}$ & $\begin{array}{c}5.07 \\
(57.29)\end{array}$ & $\begin{array}{c}5.84 \\
(34.52)\end{array}$ & $\begin{array}{c}8.37 \\
(94.58)\end{array}$ & $\begin{array}{l}10.06 \\
(59.46)\end{array}$ \\
\hline$t_{\text {mean }}$ & 0.19 & 0.28 & 0.22 & 0.32 & 0.18 & 0.28 & 0.21 & 0.33 \\
\hline$t_{\text {std }}$ & 0.92 & 0.93 & 0.86 & 0.89 & 0.94 & 0.96 & 0.88 & 0.93 \\
\hline$\frac{\text { AsySE }}{\text { EmpStd }}$ & 1.08 & 1.08 & 1.14 & 1.11 & 1.05 & 1.04 & 1.10 & 1.07 \\
\hline
\end{tabular}




\section{Table IV: C-CAPM (changing betas)}

$$
\lambda_{1}(W=I), \lambda_{2}\left(W=\Sigma_{e e}^{-1}\right) \text { vs. } \lambda^{\star}
$$

We simulate a C-CAPM economy 10,000 times under the null (the $\alpha$ parameters are set to zero). Parameter estimates of the LFM are obtained using GMM with $W=I$ for different values of $\beta$. We denote these different values of $\beta$ with $\beta_{a d j}=m * \beta$, where $m$ is a scalar. In the i.i.d bootstrap, we simulate jointly the factor and the residuals from the estimated LFM. The adjusted returns that we construct have the same volatility of the original returns. Columns two through six report, in the order, simulation results for the case of $m=1 / 3,1 / 2,1,2,3$. We investigate the properties of estimates of the LFM using the full sample of 525 observations, the ten size portfolios (size) and the two weighting matrices $W=I$ and $W=$ see $=\Sigma_{e e}^{-1}$. Estimates of the parameters of the $\mathrm{LFM}^{\star}$ are obtained by exactly-identified GMM. Asymptotic statistics are obtained assuming no serial correlation. We report the population value of the risk premium as a percentage of the population standard deviation of the excess return on the corresponding mimicking portfolio, the average value across simulations of the risk premium as a percentage of the population standard deviation of the excess return on the corresponding mimicking portfolio, absolute (percentage) bias and absolute (percentage) root mean square errors (RMSE), mean and standard deviation of the t-ratios across simulations, and the ratio between average asymptotic standard errors across simulations and empirical standard deviation. 


\begin{tabular}{|c|c|c|c|c|c|}
\hline & $m=1 / 3$ & $m=1 / 2$ & base & $\mathrm{m}=\mathbf{2}$ & $\mathrm{m}=3$ \\
\hline$\lambda_{1}$ & 11.75 & 11.72 & 11.57 & 11.00 & 10.21 \\
\hline$\hat{\lambda}_{1}$ & 10.11 & 12.98 & 12.31 & 11.19 & 10.30 \\
\hline$\lambda_{2}$ & 16.35 & 16.27 & 15.86 & 14.48 & 12.81 \\
\hline$\hat{\lambda}_{2}$ & 5.13 & 8.46 & 12.98 & 13.62 & 12.38 \\
\hline$\lambda^{\star}$ & 16.35 & 16.27 & 15.86 & 14.48 & 12.81 \\
\hline$\hat{\lambda}^{\star}$ & 16.03 & 16.07 & 15.75 & 14.36 & 12.67 \\
\hline Bias $_{\lambda_{1}}$ & $\begin{array}{l}-1.64 \\
(-13.96)\end{array}$ & $\begin{array}{c}1.26 \\
(10.75)\end{array}$ & $\begin{array}{c}0.74 \\
(6.40)\end{array}$ & $\begin{array}{c}0.19 \\
(1.73)\end{array}$ & $\begin{array}{c}0.09 \\
(0.88)\end{array}$ \\
\hline Bias $_{\lambda_{2}}$ & $\begin{array}{l}-11.22 \\
(-68.62)\end{array}$ & $\begin{array}{c}-7.81 \\
(-48.00)\end{array}$ & $\begin{array}{l}-2.88 \\
(-18.16)\end{array}$ & $\begin{array}{l}-0.85 \\
(-5.87)\end{array}$ & $\begin{array}{l}-0.43 \\
(-3.36)\end{array}$ \\
\hline $\operatorname{Bias}_{\lambda^{\star}}$ & $\begin{array}{l}-0.32 \\
(-1.96)\end{array}$ & $\begin{array}{l}-0.20 \\
(-1.23)\end{array}$ & $\begin{array}{l}-0.11 \\
(-0.69)\end{array}$ & $\begin{array}{l}-0.11 \\
(-0.76)\end{array}$ & $\begin{array}{l}-0.14 \\
(-1.10)\end{array}$ \\
\hline$R M S E_{\lambda_{1}}$ & $\begin{array}{c}13.84 \\
(117.78)\end{array}$ & $\begin{array}{c}11.18 \\
(95.39)\end{array}$ & $\begin{array}{c}6.10 \\
(52.72)\end{array}$ & $\begin{array}{c}4.74 \\
(43.09)\end{array}$ & $\begin{array}{c}4.55 \\
(44.56)\end{array}$ \\
\hline$R M S E_{\lambda_{2}}$ & $\begin{array}{c}11.81 \\
(72.23)\end{array}$ & $\begin{array}{c}8.88 \\
(54.58)\end{array}$ & $\begin{array}{c}5.28 \\
(33.29)\end{array}$ & $\begin{array}{c}4.41 \\
(30.46)\end{array}$ & $\begin{array}{c}4.36 \\
(34.04)\end{array}$ \\
\hline$R M S E_{\lambda^{\star}}$ & $\begin{array}{c}11.75 \\
(71.87)\end{array}$ & $\begin{array}{c}8.57 \\
(52.67)\end{array}$ & $\begin{array}{c}5.82 \\
(36.70)\end{array}$ & $\begin{array}{c}4.74 \\
(32.73)\end{array}$ & $\begin{array}{c}4.46 \\
(34.82)\end{array}$ \\
\hline$\left(\frac{A s y S E}{E m p S t d}\right)_{\lambda_{1}}$ & 1.19 & 1.06 & 0.97 & 0.99 & 0.99 \\
\hline$\left(\frac{A s y S E}{E m p S t d}\right)_{\lambda_{2}}$ & 0.85 & 0.93 & 0.98 & 0.98 & 0.99 \\
\hline$\left(\frac{A s y S E}{E m p S t d}\right)_{\lambda^{\star}}$ & 1.12 & 1.10 & 1.05 & 1.00 & 1.00 \\
\hline
\end{tabular}

- size portfolios, full sample, i.i.d bootstrap, 0 lags, $\beta_{a d j}=m * \beta$ 


\section{Table V: C-CAPM (Noisy Factor)}

$$
\lambda_{1}(W=I), \lambda_{2}\left(W=\Sigma_{e e}^{-1}\right) \text { vs. } \lambda^{\star}
$$

We simulate a C-CAPM economy 10,000 times under the null (the $\alpha$ parameters are set to zero). Parameter estimates of the LFM are obtained using GMM with $W=I$. In the i.i.d bootstrap, we simulate jointly the factor and the residuals from the estimated LFM. For each bootstrap replication, we add an i.i.d. Gaussian shock to the factor. The standard deviation of the shock is proportional (constant of proportionality equal to $c$ ) to the population standard deviation of the factor. Columns two through six report simulation results for the case of no noise and the case of noise $(c=0.5,1,1.5,2.0)$, respectively. We investigate the properties of estimates of the LFM using the full sample of 525 observations, the ten size portfolios (size) and the two weighting matrices $W=I$ and $W=$ see $=\Sigma_{e e}^{-1}$. Estimates of the parameters of the LFM ${ }^{\star}$ are obtained by exactlyidentified GMM. Asymptotic statistics are obtained assuming no serial correlation. We report the population value of the risk premium as a percentage of the population standard deviation of the excess return on the corresponding mimicking portfolio, the average value across simulations of the risk premium as a percentage of the population standard deviation of the excess return on the corresponding mimicking portfolio, absolute (percentage) bias and absolute (percentage) root mean square errors (RMSE), mean and standard deviation of the t-ratios across simulations, and the ratio between average asymptotic standard errors across simulations and empirical standard deviation. 


\begin{tabular}{|c|c|c|c|c|c|}
\hline & no noise & $c=0.50$ & $c=1.0$ & $c=1.50$ & $c=2.0$ \\
\hline$\lambda_{1}$ & 11.57 & 11.57 & 11.57 & 11.57 & 11.57 \\
\hline$\hat{\lambda}_{1}$ & 12.31 & 15.50 & 25.90 & 42.56 & 60.52 \\
\hline$\lambda_{2}$ & 15.86 & 15.86 & 15.86 & 15.86 & 15.86 \\
\hline$\hat{\lambda}_{2}$ & 12.98 & 15.43 & 21.44 & 28.35 & 34.40 \\
\hline$\lambda^{\star}$ & 15.86 & 15.86 & 15.86 & 15.86 & 15.86 \\
\hline$\hat{\lambda}^{\star}$ & 15.75 & 15.74 & 15.74 & 15.73 & 15.73 \\
\hline Bias $_{\lambda_{1}}$ & $\begin{array}{c}0.74 \\
(6.40)\end{array}$ & $\begin{array}{c}3.93 \\
(33.97)\end{array}$ & $\begin{array}{c}14.33 \\
(123.85)\end{array}$ & $\begin{array}{c}30.99 \\
(267.85)\end{array}$ & $\begin{array}{c}48.95 \\
(423.08)\end{array}$ \\
\hline Bias $_{\lambda_{2}}$ & $\begin{array}{l}-2.88 \\
(-18.16)\end{array}$ & $\begin{array}{l}-0.43 \\
(-2.71)\end{array}$ & $\begin{array}{c}5.58 \\
(35.18)\end{array}$ & $\begin{array}{c}12.49 \\
(78.75)\end{array}$ & $\begin{array}{c}18.54 \\
(116.90)\end{array}$ \\
\hline Biass $_{\lambda^{\star}}$ & $\begin{array}{l}-0.11 \\
(-0.69)\end{array}$ & $\begin{array}{l}-0.12 \\
(-0.76)\end{array}$ & $\begin{array}{l}-0.12 \\
(-0.76)\end{array}$ & $\begin{array}{l}-0.13 \\
(-0.82)\end{array}$ & $\begin{array}{l}-0.13 \\
(-0.82)\end{array}$ \\
\hline$R M S E_{\lambda_{1}}$ & $\begin{array}{c}6.10 \\
(52.72)\end{array}$ & $\begin{array}{c}8.87 \\
(76.66)\end{array}$ & $\begin{array}{c}21.69 \\
(187.47)\end{array}$ & $\begin{array}{c}46.62 \\
(402.94)\end{array}$ & $\begin{array}{c}79.97 \\
(691.18)\end{array}$ \\
\hline$R M S E_{\lambda_{2}}$ & $\begin{array}{c}5.28 \\
(33.29)\end{array}$ & $\begin{array}{c}5.41 \\
(34.11)\end{array}$ & $\begin{array}{c}10.09 \\
(63.62)\end{array}$ & $\begin{array}{c}17.96 \\
(113.24)\end{array}$ & $\begin{array}{c}26.28 \\
(165.70)\end{array}$ \\
\hline$R M S E_{\lambda^{\star}}$ & $\begin{array}{c}5.82 \\
(36.70)\end{array}$ & $\begin{array}{c}6.04 \\
(38.08)\end{array}$ & $\begin{array}{c}6.76 \\
(42.62)\end{array}$ & $\begin{array}{c}7.82 \\
(49.30)\end{array}$ & $\begin{array}{c}9.10 \\
(57.38)\end{array}$ \\
\hline$\left(\frac{A s y S E}{E m p S t d}\right)_{\lambda_{1}}$ & 0.97 & 0.99 & 0.99 & 1.04 & 1.10 \\
\hline$\left(\frac{A s y S E}{E m p S t d}\right)_{\lambda_{2}}$ & 0.98 & 1.00 & 1.01 & 1.00 & 0.96 \\
\hline$\left(\frac{A s y S E}{E m p S t d}\right)_{\lambda^{\star}}$ & 1.05 & 1.06 & 1.09 & 1.11 & 1.13 \\
\hline
\end{tabular}

- size portfolios, full sample, i.i.d bootstrap, 0 lags, shock $N\left(0, s_{n}^{2}\right)$ with $s_{n}=c * \sigma_{y}$ 
Table VI: C-CAPM; $\beta$ vs $\beta^{\star}$

We simulate a one-factor C-CAPM economy 10,000 times. Parameter estimates of the LFM are obtained using GMM with $W=I$. In the bootstrap (i.i.d. and block), we simulate jointly the factor and the residuals from the estimated LFM. We consider two lengths of the data set: the full sample of 525 observations (full sample) and a shorter sample of 240 observations (240 months). We consider two choices of assets, the ten size portfolios (size) and the 25 Fama-French portfolios (FF). Estimates of the parameters of the LFM ${ }^{\star}$ are obtained by exactly-identified GMM. Asymptotic statistics are obtained assuming no serial correlation in the i.i.d bootstrap and serial correlation of order 3 (Newey-West adjustment) in the block bootstrap. Both $\beta$ and $\beta^{\star}$ estimates (average $\beta$ s and $\beta^{\star}$ s across simulations and across assets) are scaled by the ratio between the population standard deviations of the factor and of the excess return. We report population and average values of the betas, absolute (percentage) bias and absolute (percentage) root mean square errors (RMSE), mean and standard deviation of the t-ratios across simulations and the ratio between average asymptotic standard errors across simulations and empirical standard deviation. 
Panel A: Finite Sample properties of $\beta$

\begin{tabular}{|l||ccc|c|c|c|c|c|c|}
\hline \multicolumn{1}{|c||}{} & \multicolumn{4}{c|}{ IID Bootstrap } & \multicolumn{4}{c|}{ Block Bootstrap } \\
\hline & Full Sample & \multicolumn{2}{c|}{ 240 Months } & Full Sample & 240 Months \\
\hline \multicolumn{1}{|c||}{ size } & FF & size & FF & Size & FF & Size & FF \\
\hline$\beta$ & 17.92 & 16.27 & 17.92 & 16.27 & 17.92 & 16.27 & 17.92 & 16.27 \\
$\hat{\beta}$ & 17.80 & 16.26 & 17.70 & 16.29 & 17.84 & 16.31 & 17.69 & 16.26 \\
Bias & -0.12 & -0.01 & -0.22 & 0.02 & -0.08 & 0.04 & -0.23 & -0.01 \\
RMSE & $(-0.67)$ & $(-0.06)$ & $(-1.23)$ & $(-0.12)$ & $(-0.45)$ & $(0.25)$ & $(-1.28)$ & $(-0.06)$ \\
$t_{\text {mean }}$ & 4.22 & 4.24 & 6.25 & 6.26 & 4.04 & 4.15 & 5.99 & 6.13 \\
$t_{\text {std }}$ & $-0.55)$ & $(26.06)$ & $(34.88)$ & $(38.48)$ & $(22.54)$ & $(25.51)$ & $(33.43)$ & $(37.68)$ \\
AsySE & 1.02 & -0.02 & -0.01 & -0.02 & -0.00 & -0.00 & -0.02 & -0.03 \\
EmpStd & 0.98 & 0.98 & 0.98 & 0.98 & 0.99 & 0.98 & 0.99 & 0.98 \\
\hline
\end{tabular}

Panel B: Finite Sample properties of $\beta^{\star}$

\begin{tabular}{|l||c|c|c|c|c|c|c|c|}
\hline \multicolumn{1}{|c||}{} & \multicolumn{4}{c|}{ IID Bootstrap } & \multicolumn{4}{c|}{ Block Bootstrap } \\
\hline \multicolumn{1}{|c||}{} & Full Sample & \multicolumn{2}{c|}{ 240 Months } & Full Sample & \multicolumn{2}{c|}{ 240 Months } \\
\hline \multicolumn{1}{|c||}{ size } & FF & size & FF & Size & FF & Size & FF \\
\hline$\beta^{\star}$ & 69.47 & 55.67 & 69.47 & 55.67 & 69.47 & 55.67 & 69.47 & 55.67 \\
$\hat{\beta}^{\star}$ & 57.29 & 37.00 & 46.50 & 26.00 & 57.63 & 37.42 & 46.73 & 26.39 \\
Bias & -12.18 & -18.67 & -22.97 & -29.67 & -11.84 & -18.25 & -22.74 & -29.28 \\
RMSE & $(-17.53)$ & $(-33.54)$ & $(-33.06)$ & $(-53.30)$ & $(-17.04)$ & $(-32.78)$ & $(-32.73)$ & $(-52.60)$ \\
$t_{\text {mean }}$ & 17.73 & 21.69 & 27.88 & 31.63 & 17.15 & 21.32 & 27.19 & 31.24 \\
$t_{\text {std }}$ & $(25.52)$ & $(38.96)$ & $(40.13)$ & $(56.82)$ & $(24.69)$ & $(38.30)$ & $(39.14)$ & $(56.12)$ \\
AsySE & -1.14 & -1.83 & -1.71 & -2.99 & -1.14 & -1.79 & -1.73 & -2.94 \\
EmpStd & 1.15 & 1.31 & 1.31 & 1.72 & 1.15 & 1.29 & 1.30 & 1.68 \\
& 1.07 & 1.11 & 1.11 & 1.10 & 1.09 & 1.12 & 1.14 & 1.12 \\
\hline
\end{tabular}


Table VII: I-CAPM; $\beta$ vs $\beta^{\star}$

We simulate a two-factor I-CAPM economy 10,000 times under the null (the $\alpha$ parameters are set to zero). The two factors are the VW NYSE/AMEX/NASDAQ index return and the change in the dividend yield. Parameter estimates of the LFM are obtained using GMM with $W=I$. In the bootstrap (i.i.d. and block), we simulate jointly the factor and the residuals from the estimated LFM. We consider two lengths of the data set: the full sample of 525 observations (full sample) and a shorter sample of 240 observations (240 months). We consider two choices of assets, the ten size portfolios (size) and the 25 Fama-French portfolios (FF). Estimates of the parameters of the $\mathrm{LFM}^{\star}$ are obtained by exactly-identified GMM. Asymptotic statistics are obtained assuming no serial correlation in the i.i.d bootstrap and serial correlation of order 3 (Newey-West adjustment) in the block bootstrap. Both $\beta$ and $\beta^{\star}$ estimates (average $\beta$ s and $\beta^{\star}$ s across simulations and across assets) are scaled by the ratio between the population standard deviations of the factor and of the excess return. We report population and average values of the betas, absolute (percentage) bias and absolute (percentage) root mean square errors (RMSE), mean and standard deviation of the t-ratios across simulations and the ratio between average asymptotic standard errors across simulations and empirical standard deviation. 
Panel A: Finite Sample properties of $\beta$

\begin{tabular}{|c|c|c|c|c|c|c|c|c|}
\hline & \multicolumn{4}{|c|}{ IID Bootstrap } & \multicolumn{4}{|c|}{ Block Bootstrap } \\
\hline & \multicolumn{2}{|c|}{ Full Sample } & \multicolumn{2}{|c|}{240 Months } & \multicolumn{2}{|c|}{ Full Sample } & \multicolumn{2}{|c|}{240 Months } \\
\hline & size & FF & size & FF & Size & FF & Size & FF \\
\hline$\beta$ & -11.81 & -7.77 & -11.81 & -7.77 & -11.81 & -7.77 & -11.81 & -7.77 \\
\hline$\hat{\beta}$ & -11.82 & -7.98 & -11.86 & -8.02 & -11.87 & -8.03 & -12.00 & -8.16 \\
\hline Bias & $\begin{array}{c}-0.01 \\
(-0.08)\end{array}$ & $\begin{array}{c}-0.22 \\
(-2.83)\end{array}$ & $\begin{array}{c}-0.05 \\
(-0.42)\end{array}$ & $\begin{array}{c}-0.25 \\
(-3.22)\end{array}$ & $\begin{array}{c}-0.12 \\
(-1.02)\end{array}$ & $\begin{array}{l}-0.06 \\
(-0.77)\end{array}$ & $\begin{array}{c}-0.19 \\
(-1.61)\end{array}$ & $\begin{array}{c}-0.39 \\
(-5.02)\end{array}$ \\
\hline$R M S E$ & $\begin{array}{c}3.77 \\
(31.92)\end{array}$ & $\begin{array}{c}3.70 \\
(47.62)\end{array}$ & $\begin{array}{c}5.64 \\
(47.76)\end{array}$ & $\begin{array}{c}5.50 \\
(70.79)\end{array}$ & $\begin{array}{c}3.73 \\
(31.58)\end{array}$ & $\begin{array}{c}3.67 \\
(47.23)\end{array}$ & $\begin{array}{c}5.60 \\
(47.42)\end{array}$ & $\begin{array}{c}5.47 \\
(70.40)\end{array}$ \\
\hline$t_{\text {mean }}$ & 0.04 & -0.02 & 0.05 & -0.00 & 0.03 & -0.03 & 0.04 & -0.02 \\
\hline$t_{\text {std }}$ & 1.02 & 1.01 & 1.05 & 1.03 & 1.03 & 1.03 & 1.06 & 1.06 \\
\hline$\frac{\text { AsySE }}{\text { EmpStd }}$ & 0.99 & 0.99 & 0.97 & 0.97 & 0.98 & 0.98 & 0.96 & 0.96 \\
\hline
\end{tabular}

Panel B: Finite Sample properties of $\beta^{\star}$

\begin{tabular}{|c|c|c|c|c|c|c|c|c|}
\hline & \multicolumn{4}{|c|}{ IID Bootstrap } & \multicolumn{4}{|c|}{ Block Bootstrap } \\
\hline & \multicolumn{2}{|c|}{ Full Sample } & \multicolumn{2}{|c|}{240 Months } & \multicolumn{2}{|c|}{ Full Sample } & \multicolumn{2}{|c|}{240 Months } \\
\hline & size & FF & size & FF & Size & FF & Size & FF \\
\hline$\beta^{\star}$ & -57.18 & -27.51 & -57.18 & -27.51 & -57.18 & -27.51 & -57.18 & -27.51 \\
\hline$\hat{\beta}^{\star}$ & -47.29 & -21.65 & -38.31 & -16.61 & -47.29 & -21.86 & -38.47 & -16.87 \\
\hline Bias & $\begin{array}{c}9.89 \\
(17.30)\end{array}$ & $\begin{array}{c}5.86 \\
(21.30)\end{array}$ & $\begin{array}{c}18.87 \\
(33.00)\end{array}$ & $\begin{array}{c}10.90 \\
(39.62)\end{array}$ & $\begin{array}{c}9.89 \\
(17.30)\end{array}$ & $\begin{array}{c}5.65 \\
(20.54)\end{array}$ & $\begin{array}{c}18.71 \\
(32.72)\end{array}$ & $\begin{array}{c}10.64 \\
(38.68)\end{array}$ \\
\hline$R M S E$ & $\begin{array}{c}18.57 \\
(32.48)\end{array}$ & $\begin{array}{c}12.51 \\
(45.47)\end{array}$ & $\begin{array}{c}28.85 \\
(50.45)\end{array}$ & $\begin{array}{c}17.82 \\
(64.78)\end{array}$ & $\begin{array}{c}18.39 \\
(32.16)\end{array}$ & $\begin{array}{l}12.30 \\
(44.71)\end{array}$ & $\begin{array}{c}28.46 \\
(49.77)\end{array}$ & $\begin{array}{c}17.56 \\
(63.83)\end{array}$ \\
\hline$t_{\text {mean }}$ & 0.83 & 0.65 & 1.29 & 1.05 & 0.85 & 0.65 & 1.32 & 1.08 \\
\hline$t_{s t d}$ & 1.09 & 1.04 & 1.23 & 1.13 & 1.10 & 1.05 & 1.27 & 1.17 \\
\hline$\frac{A s y S E}{E m p S t d}$ & 1.08 & 1.03 & 1.14 & 1.03 & 1.08 & 1.02 & 1.13 & 1.01 \\
\hline
\end{tabular}




\section{Table VIII: Size of the Wald Test (C-CAPM)}

We simulate a one-factor C-CAPM economy 10,000 times under the null (the $\alpha$ parameters are set to zero). Parameter estimates of the LFM are obtained using GMM with $W=I$. In the bootstrap (i.i.d. and block), we simulate jointly the factor and the residuals from the estimated LFM. We consider two lengths of the data set: the full sample of 525 observations (full sample) and a shorter sample of 240 observations (240 months). We consider two choices of assets, the ten size portfolios (size) and the 25 Fama-French portfolios (FF). When we investigate the properties of estimates of the LFM, we consider estimates based on the two weighting matrices $W=I$ and $W=s e e=\Sigma_{e e}^{-1}$. Estimates of the parameters of the $\mathrm{LFM}^{\star}$ are obtained by exactly-identified GMM. Asymptotic statistics are obtained assuming no serial correlation in the i.i.d bootstrap and serial correlation of order 3 (Newey-West adjustment) in the block bootstrap. We report the theoretical and actual sizes of the Wald test for the one-factor model. Panels A and B report results for the LFM specification, for the full sample and for the short sample of 240 observations, respectively. Panel C reports results for the $\mathrm{LFM}^{\star}$ specification. The LFM test statistic should be distributed $\chi_{N-K}^{2}$, while the LFM test statistic should be distributed $\chi_{N}^{2}$. 
Panel A: LFM (IID Bootstrap)

\begin{tabular}{|l||r|r|r|r|r|r|r|r|}
\hline \multicolumn{1}{|c||}{} & \multicolumn{4}{c|}{ Full Sample } & \multicolumn{4}{c|}{ 240 Months } \\
\hline \multicolumn{1}{|c||}{} & \multicolumn{2}{|c|}{ size } & \multicolumn{2}{c|}{ FF } & \multicolumn{2}{|c|}{ size } & \multicolumn{2}{c|}{ FF } \\
\hline \multicolumn{1}{|c|}{ W=I } & W=sse & W=I & W=sse & W=I & W=sse & W=I & W=sse \\
\hline Ave. Chisq & 8.47 & 9.57 & 23.61 & 27.54 & 8.05 & 9.79 & 23.01 & 27.48 \\
0.50 & 0.43 & 0.54 & 0.47 & 0.67 & 0.40 & 0.57 & 0.46 & 0.68 \\
0.25 & 0.21 & 0.30 & 0.24 & 0.42 & 0.19 & 0.31 & 0.23 & 0.43 \\
0.10 & 0.09 & 0.14 & 0.11 & 0.23 & 0.08 & 0.14 & 0.10 & 0.22 \\
0.05 & 0.05 & 0.07 & 0.06 & 0.15 & 0.04 & 0.08 & 0.05 & 0.13 \\
0.025 & 0.027 & 0.040 & 0.034 & 0.092 & 0.022 & 0.044 & 0.027 & 0.075 \\
0.01 & 0.01 & 0.02 & 0.01 & 0.05 & 0.01 & 0.02 & 0.01 & 0.04 \\
\hline
\end{tabular}

Panel B: LFM (Block Bootstrap)

\begin{tabular}{|l||rr|r|r|r|r|r|r|}
\hline \multicolumn{1}{|c||}{} & \multicolumn{4}{c|}{ Full Sample } & \multicolumn{4}{c|}{ 240 Months } \\
\hline \multicolumn{1}{|c}{} & \multicolumn{2}{|c}{ size } & \multicolumn{2}{c|}{ FF } & \multicolumn{2}{|c|}{ size } & \multicolumn{3}{c|}{ FF } \\
\hline \multicolumn{1}{|c||}{ W=I } & W=sse & W=I & W=sse & W=I & W=sse & W=I & W=sse \\
\hline Ave. Chisq & 8.08 & 9.10 & 22.43 & 25.77 & 7.52 & 9.09 & 20.76 & 24.18 \\
0.50 & 0.40 & 0.51 & 0.41 & 0.60 & 0.35 & 0.52 & 0.33 & 0.55 \\
0.25 & 0.18 & 0.25 & 0.18 & 0.34 & 0.15 & 0.26 & 0.11 & 0.24 \\
0.10 & 0.07 & 0.10 & 0.07 & 0.15 & 0.05 & 0.10 & 0.02 & 0.06 \\
0.05 & 0.03 & 0.06 & 0.03 & 0.08 & 0.02 & 0.05 & 0.01 & 0.02 \\
0.025 & 0.017 & 0.028 & 0.015 & 0.042 & 0.010 & 0.020 & 0.002 & 0.006 \\
0.01 & 0.01 & 0.01 & 0.00 & 0.02 & 0.00 & 0.01 & 0.00 & 0.00 \\
\hline
\end{tabular}

Panel C: LFM ${ }^{\star}$

\begin{tabular}{|l||rr|r|c|r|r|r|r|}
\hline \multicolumn{1}{|c||}{} & \multicolumn{4}{c|}{ IID Bootstrap } & \multicolumn{4}{c|}{ Block Bootstrap } \\
\hline & Full Sample & \multicolumn{2}{c|}{ 240 Months } & \multicolumn{1}{c|}{ Full Sample } & 240 Months \\
\hline \multicolumn{1}{|c||}{} & size & FF & size & FF & size & FF & size & FF \\
\hline \multirow{2}{*}{ Ave. Chisq } & 12.01 & 33.54 & 11.94 & 33.59 & 12.05 & 35.48 & 12.38 & 38.72 \\
0.50 & 0.63 & 0.82 & 0.63 & 0.81 & 0.63 & 0.85 & 0.65 & 0.88 \\
0.25 & 0.40 & 0.62 & 0.39 & 0.62 & 0.40 & 0.68 & 0.42 & 0.74 \\
0.10 & 0.21 & 0.42 & 0.21 & 0.42 & 0.21 & 0.49 & 0.23 & 0.58 \\
0.05 & 0.14 & 0.31 & 0.13 & 0.31 & 0.13 & 0.38 & 0.15 & 0.47 \\
0.025 & 0.082 & 0.227 & 0.080 & 0.227 & 0.087 & 0.287 & 0.099 & 0.388 \\
0.01 & 0.04 & 0.15 & 0.04 & 0.15 & 0.05 & 0.20 & 0.06 & 0.30 \\
\hline
\end{tabular}

$\mathbf{s s e}=\hat{\Sigma}_{e e}^{-1}$ 


\section{Table IX: Size of the Wald Test (I-CAPM)}

We simulate a two-factor I-CAPM economy 10,000 times under the null (the $\alpha$ parameters are set to zero). The two factors are the VW NYSE/AMEX/NASDAQ index return and the change in the dividend yield. Parameter estimates of the LFM are obtained using GMM with $W=I$. Parameter estimates of the LFM are obtained using GMM with $W=I$. In the bootstrap (i.i.d. and block), we simulate jointly the factor and the residuals from the estimated LFM. We consider two lengths of the data set: the full sample of 525 observations (full sample) and a shorter sample of 240 observations (240 months). We consider two choices of assets, the ten size portfolios (size) and the 25 Fama-French portfolios (FF). When we investigate the properties of estimates of the LFM, we consider estimates based on the two weighting matrices $W=I$ and $W=s e e=\Sigma_{e e}^{-1}$. Estimates of the parameters of the $\mathrm{LFM}^{\star}$ are obtained by exactly-identified GMM. Asymptotic statistics are obtained assuming no serial correlation in the i.i.d bootstrap and serial correlation of order 3 (Newey-West adjustment) in the block bootstrap. We report the theoretical and actual sizes of the Wald test for the two-factor model. Panels A and B report results for the LFM specification, for the full sample and for the short sample of 240 observations, respectively. Panel C reports results for the LFM^ specification. The LFM test statistic should be distributed $\chi_{N-K_{2}}^{2}$, where $K_{2}=1$ represents the number of non-traded factors, while the LFM test statistic should be distributed $\chi_{N}^{2}$. 
Panel A: LFM (IID Bootstrap)

\begin{tabular}{|l||rr|r|r|r|r|r|r|}
\hline \multicolumn{1}{|c||}{} & \multicolumn{4}{c|}{ Full Sample } & \multicolumn{4}{c|}{ 240 Months } \\
\hline \multicolumn{1}{|c||}{} & \multicolumn{2}{c|}{ size } & \multicolumn{2}{c|}{ FF } & \multicolumn{2}{|c|}{ size } & \multicolumn{2}{c|}{ FF } \\
\hline \multicolumn{1}{|c||}{ W=I } & W=sse & W=I & W=sse & W=I & W=sse & W=I & W=sse \\
\hline \multirow{2}{*}{ Ave. Chisq } & 8.66 & 9.27 & 24.25 & 26.44 & 8.28 & 9.30 & 24.39 & 27.00 \\
0.50 & 0.46 & 0.52 & 0.50 & 0.62 & 0.43 & 0.52 & 0.52 & 0.66 \\
0.25 & 0.22 & 0.27 & 0.27 & 0.37 & 0.20 & 0.28 & 0.28 & 0.41 \\
0.10 & 0.09 & 0.12 & 0.13 & 0.19 & 0.07 & 0.12 & 0.13 & 0.20 \\
0.05 & 0.04 & 0.06 & 0.07 & 0.11 & 0.04 & 0.06 & 0.07 & 0.11 \\
0.025 & 0.021 & 0.033 & 0.040 & 0.062 & 0.016 & 0.030 & 0.036 & 0.061 \\
0.01 & 0.01 & 0.01 & 0.02 & 0.03 & 0.01 & 0.01 & 0.01 & 0.03 \\
\hline
\end{tabular}

Panel B: LFM (Block Bootstrap)

\begin{tabular}{|l||rr|rr|r|rr|r|r|}
\hline \multicolumn{1}{|c||}{} & \multicolumn{4}{c|}{ Full Sample } & \multicolumn{4}{c|}{ 240 Months } \\
\hline \multicolumn{1}{|c}{} & \multicolumn{2}{|c}{ size } & \multicolumn{2}{c|}{ FF } & \multicolumn{2}{|c|}{ size } & \multicolumn{3}{c|}{ FF } \\
\hline \multicolumn{1}{|c||}{ W=I } & W=sse & W=I & W=sse & W=I & W=sse & W=I & W=sse \\
\hline Ave. Chisq & 8.22 & 8.76 & 23.18 & 24.97 & 7.84 & 8.70 & 22.16 & 24.06 \\
0.50 & 0.42 & 0.48 & 0.45 & 0.56 & 0.39 & 0.48 & 0.40 & 0.53 \\
0.25 & 0.18 & 0.23 & 0.21 & 0.29 & 0.16 & 0.22 & 0.16 & 0.23 \\
0.10 & 0.06 & 0.09 & 0.09 & 0.13 & 0.05 & 0.08 & 0.04 & 0.06 \\
0.05 & 0.03 & 0.04 & 0.05 & 0.06 & 0.02 & 0.03 & 0.01 & 0.02 \\
0.025 & 0.013 & 0.020 & 0.022 & 0.031 & 0.008 & 0.014 & 0.004 & 0.007 \\
0.01 & 0.01 & 0.01 & 0.01 & 0.01 & 0.00 & 0.00 & 0.00 & 0.00 \\
\hline
\end{tabular}

Panel C: LFM ${ }^{\star}$

\begin{tabular}{|l||rr|r|c|r|r|r|r|}
\hline \multicolumn{1}{|c||}{} & \multicolumn{4}{c|}{ IID Bootstrap } & \multicolumn{4}{c|}{ Block Bootstrap } \\
\hline & Full Sample & \multicolumn{2}{c|}{ 240 Months } & \multicolumn{1}{c|}{ Full Sample } & 240 Months \\
\hline \multicolumn{1}{|c||}{} & size & FF & size & FF & size & FF & size & FF \\
\hline \multirow{2}{*}{ Ave. Chisq } & 10.87 & 32.26 & 10.87 & 33.40 & 10.75 & 34.27 & 11.24 & 38.96 \\
0.50 & 0.56 & 0.78 & 0.55 & 0.81 & 0.55 & 0.82 & 0.57 & 0.89 \\
0.25 & 0.32 & 0.58 & 0.32 & 0.62 & 0.31 & 0.64 & 0.34 & 0.75 \\
0.10 & 0.16 & 0.37 & 0.16 & 0.42 & 0.15 & 0.44 & 0.17 & 0.59 \\
0.05 & 0.09 & 0.27 & 0.09 & 0.31 & 0.09 & 0.33 & 0.11 & 0.48 \\
0.025 & 0.051 & 0.187 & 0.051 & 0.225 & 0.051 & 0.247 & 0.067 & 0.392 \\
0.01 & 0.03 & 0.11 & 0.02 & 0.14 & 0.03 & 0.17 & 0.03 & 0.30 \\
\hline
\end{tabular}

$\mathbf{s s e}=\hat{\Sigma}_{e e}^{-1}$ 


\section{Table X: Power of the Wald Test (C-CAPM)}

We simulate a one-factor C-CAPM economy 10,000 times under the alternative $(\alpha \neq 0)$. Parameter estimates of the LFM are obtained using GMM with $W=I$. In the bootstrap (i.i.d. and block), we simulate jointly the factor and the residuals from the estimated LFM. We consider two lengths of the data set: the full sample of 525 observations (full sample) and a shorter sample of 240 observations (240 months). We consider two choices of assets, the ten size portfolios (size) and the 25 Fama-French portfolios (FF). When we investigate the properties of estimates of the LFM, we consider estimates based on the two weighting matrices $W=I$ and $W=$ see $=\Sigma_{e e}^{-1}$. Estimates of the parameters of the $\mathrm{LFM}^{\star}$ are obtained by exactly-identified GMM. Asymptotic statistics are obtained assuming no serial correlation in the i.i.d bootstrap and serial correlation of order 3 (Newey-West adjustment) in the block bootstrap. We report rejection rates for the Wald test under the alternative, where the size is adjusted using the bootstrap results of Table VII. We compute the $10 \%, 5 \%$, and $1 \%$ quantiles of the empirical distribution of the Wald statistic under the null. We then compute the percentage of times the Wald statistic exceeds the corresponding quantile, when the economy is simulated under the alternative. Panels A and B report results for the LFM specification, for the full sample and for the short sample of 240 observations, respectively. Panel C reports results for the $\mathrm{LFM}^{\star}$ specification. 
Panel A: LFM (IID Bootstrap)

\begin{tabular}{|l||rr|r|r|r|r|r|r|}
\hline \multicolumn{1}{|c||}{} & \multicolumn{4}{c|}{ Full Sample } & \multicolumn{4}{c|}{ 240 Months } \\
\hline \multicolumn{1}{|c||}{} & \multicolumn{2}{c|}{ size } & \multicolumn{2}{c|}{ FF } & \multicolumn{2}{|c|}{ size } & \multicolumn{2}{c|}{ FF } \\
\hline \multicolumn{1}{|c|}{ W=I } & W=sse & W=I & W=sse & W=I & W=sse & W=I & W=sse \\
\hline Ave. Chisq & 24.11 & 32.35 & 83.78 & 104.85 & 14.48 & 19.26 & 48.85 & 58.80 \\
0.10 & 0.89 & 0.97 & 1.00 & 1.00 & 0.50 & 0.64 & 0.88 & 0.98 \\
0.05 & 0.81 & 0.93 & 0.99 & 1.00 & 0.35 & 0.50 & 0.82 & 0.95 \\
0.01 & 0.57 & 0.80 & 0.98 & 1.00 & 0.15 & 0.25 & 0.66 & 0.83 \\
\hline
\end{tabular}

Panel B: LFM (Block Bootstrap)

\begin{tabular}{|l||rr|r|r|r|r|r|r|}
\hline \multicolumn{1}{|c||}{} & \multicolumn{4}{c|}{ Full Sample } & \multicolumn{4}{c|}{ 240 Months } \\
\hline \multicolumn{1}{|c||}{} & \multicolumn{2}{c|}{ size } & \multicolumn{2}{c|}{ FF } & \multicolumn{2}{|c|}{ size } & \multicolumn{3}{c|}{ FF } \\
\hline \multicolumn{1}{|c|}{ W=I } & W=sse & W=I & W=sse & W=I & W=sse & W=I & W=sse \\
\hline Ave. Chisq & 22.56 & 28.66 & 63.73 & 75.67 & 13.17 & 16.73 & 35.82 & 41.21 \\
0.10 & 0.92 & 0.97 & 1.00 & 1.00 & 0.50 & 0.63 & 0.84 & 0.95 \\
0.05 & 0.84 & 0.93 & 0.99 & 1.00 & 0.36 & 0.49 & 0.77 & 0.90 \\
0.01 & 0.59 & 0.78 & 0.98 & 1.00 & 0.13 & 0.22 & 0.56 & 0.71 \\
\hline
\end{tabular}

Panel C: LFM $^{\star}$

\begin{tabular}{|l||rr|r|c|r|r|r|r|}
\hline \multicolumn{1}{|c||}{} & \multicolumn{4}{c|}{ IID Bootstrap } & \multicolumn{4}{c|}{ Block Bootstrap } \\
\hline & Full Sample & \multicolumn{2}{c|}{ 240 Months } & Full Sample & 240 Months \\
\hline \multicolumn{1}{|||}{} & size & FF & size & FF & size & FF & size & FF \\
\hline \multirow{2}{*}{ Ave. Chisq } & 38.27 & 138.67 & 23.21 & 83.14 & 38.35 & 143.06 & 24.07 & 93.23 \\
0.10 & 0.96 & 1.00 & 0.62 & 0.98 & 0.96 & 1.00 & 0.60 & 0.95 \\
0.05 & 0.92 & 1.00 & 0.48 & 0.96 & 0.91 & 1.00 & 0.45 & 0.90 \\
0.01 & 0.78 & 1.00 & 0.22 & 0.84 & 0.72 & 1.00 & 0.19 & 0.71 \\
\hline
\end{tabular}

sse $=\hat{\Sigma}_{e e}^{-1}$ 


\section{Table XI: Power of the Wald Test (I-CAPM)}

We simulate a two-factor I-CAPM economy 10,000 times under the alternative $(\alpha \neq 0)$. The two factors are the VW NYSE/AMEX/NASDAQ index return and the change in the dividend yield. Parameter estimates of the LFM are obtained using GMM with $W=I$. In the bootstrap (i.i.d. and block), we simulate jointly the factor and the residuals from the estimated LFM. We consider two lengths of the data set: the full sample of 525 observations (full sample) and a shorter sample of 240 observations (240 months). We consider two choices of assets, the ten size portfolios (size) and the 25 Fama-French portfolios (FF). When we investigate the properties of estimates of the LFM, we consider estimates based on the two weighting matrices $W=I$ and $W=$ see $=\Sigma_{e e}^{-1}$. Estimates of the parameters of the $\mathrm{LFM}^{\star}$ are obtained by exactly-identified GMM. Asymptotic statistics are obtained assuming no serial correlation in the i.i.d bootstrap and serial correlation of order 3 (Newey-West adjustment) in the block bootstrap. We report rejection rates for the Wald test under the alternative, where the size is adjusted using the bootstrap results of Table VIII. We compute the $10 \%, 5 \%$, and $1 \%$ quantiles of the empirical distribution of the Wald statistic under the null. We then compute the percentage of times the Wald statistic exceeds the corresponding quantile, when the economy is simulated under the alternative. Panels A and B report results for the LFM specification, for the full sample and for the short sample of 240 observations, respectively. Panel C reports results for the $\mathrm{LFM}^{\star}$ specification. 
Panel A: LFM (IID Bootstrap)

\begin{tabular}{|l||rr|r|r|r|r|r|r|}
\hline \multicolumn{1}{|c||}{} & \multicolumn{4}{c|}{ Full Sample } & \multicolumn{4}{c|}{ 240 Months } \\
\hline \multicolumn{1}{|c||}{} & \multicolumn{2}{c|}{ size } & \multicolumn{2}{c|}{ FF } & \multicolumn{2}{|c|}{ size } & \multicolumn{2}{c|}{ FF } \\
\hline \multicolumn{1}{|c|}{ W=I } & W=sse & W=I & W=sse & W=I & W=sse & W=I & W=sse \\
\hline Ave. Chisq & 25.69 & 27.91 & 92.29 & 101.28 & 15.64 & 17.36 & 52.25 & 57.12 \\
0.10 & 0.91 & 0.91 & 1.00 & 1.00 & 0.57 & 0.58 & 0.93 & 0.98 \\
0.05 & 0.85 & 0.85 & 1.00 & 1.00 & 0.43 & 0.44 & 0.89 & 0.96 \\
0.01 & 0.69 & 0.68 & 1.00 & 1.00 & 0.22 & 0.23 & 0.74 & 0.84 \\
\hline
\end{tabular}

Panel B: LFM (Block Bootstrap)

\begin{tabular}{|l||rr|r|r|r|r|r|r|}
\hline \multicolumn{1}{|c||}{} & \multicolumn{4}{c|}{ Full Sample } & \multicolumn{4}{c|}{ 240 Months } \\
\hline \multicolumn{1}{|c||}{} & \multicolumn{2}{c|}{ size } & \multicolumn{2}{c|}{ FF } & \multicolumn{2}{|c|}{ size } & \multicolumn{3}{c|}{ FF } \\
\hline \multicolumn{1}{|c|}{ W=I } & W=sse & W=I & W=sse & W=I & W=sse & W=I & W=sse \\
\hline Ave. Chisq & 23.57 & 25.37 & 69.18 & 74.25 & 14.16 & 15.51 & 38.05 & 40.67 \\
0.10 & 0.92 & 0.92 & 1.00 & 1.00 & 0.57 & 0.59 & 0.90 & 0.95 \\
0.05 & 0.87 & 0.86 & 1.00 & 1.00 & 0.44 & 0.46 & 0.82 & 0.90 \\
0.01 & 0.67 & 0.69 & 1.00 & 1.00 & 0.21 & 0.22 & 0.59 & 0.65 \\
\hline
\end{tabular}

Panel C: LFM $^{\star}$

\begin{tabular}{|l||rr|r|c|c|c|c|c|}
\hline \multicolumn{1}{|c||}{} & \multicolumn{4}{c|}{ IID Bootstrap } & \multicolumn{4}{c|}{ Block Bootstrap } \\
\hline & Full Sample & \multicolumn{2}{c|}{ 240 Months } & Full Sample & 240 Months \\
\hline \multicolumn{1}{|||}{} & size & FF & size & FF & size & FF & size & FF \\
\hline \multirow{2}{*}{ Ave. Chisq } & 33.28 & 131.03 & 20.94 & 79.05 & 33.89 & 136.00 & 22.12 & 88.97 \\
0.10 & 0.93 & 1.00 & 0.59 & 0.98 & 0.93 & 1.00 & 0.59 & 0.94 \\
0.05 & 0.88 & 1.00 & 0.47 & 0.95 & 0.88 & 1.00 & 0.47 & 0.88 \\
0.01 & 0.71 & 1.00 & 0.23 & 0.83 & 0.70 & 1.00 & 0.22 & 0.63 \\
\hline
\end{tabular}

sse $=\hat{\Sigma}_{e e}^{-1}$ 